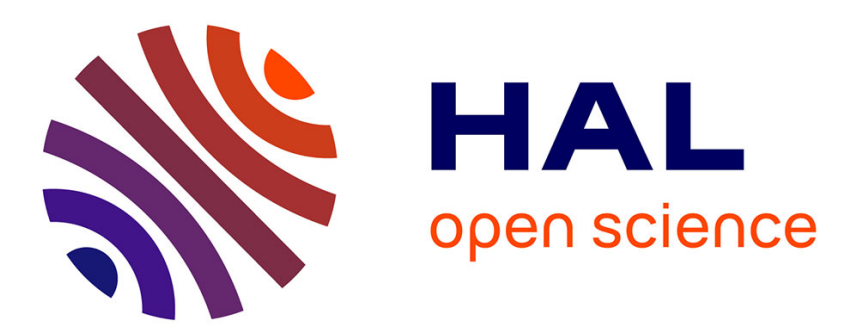

\title{
Spectral and condition number estimates of the acoustic single-layer operator for low-frequency multiple scattering in dilute media
}

Xavier Antoine, Bertrand Thierry

\section{- To cite this version:}

Xavier Antoine, Bertrand Thierry. Spectral and condition number estimates of the acoustic singlelayer operator for low-frequency multiple scattering in dilute media. Computer Methods in Applied Mechanics and Engineering, 2013, 265, pp.242-256. 10.1016/j.cma.2012.04.017 . hal-00755645

\section{HAL Id: hal-00755645 \\ https://hal.science/hal-00755645}

Submitted on 21 Nov 2012

HAL is a multi-disciplinary open access archive for the deposit and dissemination of scientific research documents, whether they are published or not. The documents may come from teaching and research institutions in France or abroad, or from public or private research centers.
L'archive ouverte pluridisciplinaire HAL, est destinée au dépôt et à la diffusion de documents scientifiques de niveau recherche, publiés ou non, émanant des établissements d'enseignement et de recherche français ou étrangers, des laboratoires publics ou privés. 


\title{
Spectral and condition number estimates of the acoustic single-layer operator for low-frequency multiple scattering in dilute media*
}

\author{
Xavier Antoine $\quad$ Bertrand Thierry ${ }^{\dagger}$
}

\begin{abstract}
The aim of this paper is to develop an analysis of the distribution of the eigenvalues of the acoustic single-layer potential for various low frequency two-dimensional multiple scattering problems. The obstacles are supposed to be distant (dilute media). In [26], it is shown that an approach based on the Gershgorin disks provides limited spectral information. We therefore introduce an alternative approach by applying the power iteration method to the limit matrix (associated with the zero order spatial modes) which results in satisfactory estimates. All these approximations are built for circular cylinders and formally extended to ellipses and rectangles for linear boundary element methods with non uniform meshes. This study is completed in [7] by spectral estimates for the case of close obstacles.
\end{abstract}

\section{Introduction}

We propose some spectral and condition number estimates of the acoustic single-layer potential for low frequency two-dimensional multiple scattering configurations. A few studies have already been developed for acoustic integral operators but for (high frequency) single scattering problems. For examples, let us mention the circular cylinder case $[5,6,20,21]$, the case of various convex and non convex single scatterers [9, 11] or also the case of open surfaces and guiding structures [2, 3, 14]. Considering multiple scattering leads to new difficulties due to the presence of interactions between scatterers [22]. Mathematically, this means that new parameters are involved into the spectral analysis: the distances between separated obstacles. We focus here on the low frequency case because the medium and high frequency regimes are still out of reach and first require a better understanding of the low frequency situation that we investigate. The single-layer potential is considered as an example but other standard integral operators (mainly the trace of the doublelayer potential and the normal derivative traces of the single- and double-layer potentials) could also be studied by using a similar strategy. This would lead to the possibility of obtaining spectral properties for examples of the Combined Field Integral Equation (CFIE) [8, 19] and BrakhageWerner integral equation formulations $[8,10]$, as well as their recent improved versions $[5,6,16]$, for multiple scattering problems. These integral equations are widely used for computational purposes since they are well-posed at any frequency $[15,23]$. Furthermore, for single scattering configurations, they are known to have a better conditioning than the Electric Field Integral Equation (EFIE) [8]

\footnotetext{
${ }^{*}$ The authors are partially supported by the French ANR fundings under the project MicroWave NT09_460489.

${ }^{\dagger}$ Institut Elie Cartan Nancy, Université de Lorraine, CNRS UMR 7502, INRIA CORIDA Team, Boulevard des Aiguillettes B.P. 239, F-54506 Vandoeuvre-lès-Nancy, France (Xavier.Antoine@iecn.u-nancy.fr, Bertrand.Thierry@iecn.u-nancy.fr).
} 
or Magnetic Field Integral Equation (MFIE) [8] which may have interior spurious resonances [16]. Let us mention here that deriving spectral properties of an integral equation is a central step for a better understanding of the convergence behavior of iterative Krylov subspace solvers [24] which are generally coupled with a Fast Multipole Method [13, 17]. Indeed, the distribution of the eigenvalues of the integral equation to solve reflects the convergence properties $[24,25]$ where eigenvalue clustering is expected. For this reason, analyzing the spectral information of basic integral operators is important. In particular, it is crucial to understand how the physical (wave number, geometry) and (boundary element) discretization parameters impacts the convergence behavior. The reason why we focus our study on the single-layer potential at low frequency is that it already requires a non trivial and tricky asymptotic analysis. In particular, several regimes must be analyzed in details. We propose here to examine the case of dilute media which corresponds to the situation where the distances between the obstacles are larger than a few wavelengths. In [7], we develop a complete analysis when the obstacles are close (dense media). The intermediate case corresponding to distances between the obstacles that are of the order of the wavelength appears to be more delicate. Concerning the numerical solution of multiple scattering problems, let us note for completeness that other computational solutions can be considered based on purely analytical or semi-analytical solutions $[4,12,22]$ or even with suitable non-reflecting boundary conditions well-adapted to the geometrical configuration [18].

A first step of our approach consists in a detailed analysis of the eigenvalues distribution of the acoustic single-layer operator for single scattering in the low frequency regime (section 2). We investigate the case of a disk since the integral operator can be diagonalized in the Fourier basis. We obtain asymptotic expressions of the eigenvalues of the operator which are then extended to other obstacles (elliptical and rectangular cylinders) and to the linear boundary element approximation of the integral operator. In Section 3, we give the expression of the single-layer operator for multiple scattering by a collection of circular cylinders. Unlike the single scattering case, the operator is not diagonal. For this reason, a more complicate asymptotic analysis must be developed. In [26], we studied the possibility of applying Gershgorin disks theorems to get information on the spectrum in conjunction with asymptotic expansions for distant obstacles. It unfortunately appears that this method is quite limited. For this reason, an alternative approach based on the application of the power iteration method to a small matrix associated with the zero order Fourier modes and asymptotic expansions is proposed. We show that relatively accurate estimates of the spectrum as well as condition number of the single-layer operator can be obtained. We extend these estimates to ellipses and rectangles and to the linear boundary element approach. All these approximate formulas are validated with various numerical simulations. A conclusion is given in Section 4 .

\section{Spectral estimates for low frequency single scattering}

Let us consider a homogeneous acoustic medium filling the whole space $\mathbb{R}^{2}$ and containing a bounded open set $\Omega$ with boundary $\Gamma:=\partial \Omega$. The propagation domain $\Omega^{+}=\mathbb{R}^{2} \backslash \bar{\Omega}$ is assumed to be connected. For a real positive wave number $k$, we define the single-layer integral operator $L$ by

$$
L \rho(\mathbf{x})=\int_{\Gamma} G(\mathbf{x}, \mathbf{y}) \rho(\mathbf{y}) \mathrm{d} \Gamma(\mathbf{y}),
$$

where the Green's function $G(\cdot, \cdot)$ is given by: $\forall \mathbf{x}, \mathbf{y} \in \mathbb{R}^{2}, \mathbf{x} \neq \mathbf{y}, G(\mathbf{x}, \mathbf{y})=\frac{i}{4} H_{0}^{(1)}(k\|\mathbf{x}-\mathbf{y}\|)$, with $H_{0}^{(1)}$ the zeroth order Hankel function of the first kind, $k>0$ the real and positive wavenumber and 
$\|\mathbf{x}\|=\left(x_{1}^{2}+x_{2}^{2}\right)^{1 / 2}$. It is well-known (see e.g. Theorem 3.4.1 of [23]) that $L$ defines an isomorphism from $H^{-1 / 2}(\Gamma)$ to $H^{1 / 2}(\Gamma)$ except for the set $F_{D}(\Omega)$ of the Dirichlet irregular frequencies, that is the wave numbers $k$ for which the interior homogeneous Dirichlet problem

$$
\begin{cases}\left(\Delta+k^{2}\right) w=0 & \text { in } \Omega \\ w=0 & \text { on } \Gamma,\end{cases}
$$

admits non trivial solution. In the sequel, we assume that $k \notin F_{D}(\Omega)$. The goal of the paper consists in obtaining spectral information (eigenvalues and conditioning) of the single-layer potential for multiple scattering problems. Before this, let us consider the low frequency single scattering case.

\subsection{The circular cylinder case}

We assume here that $\Omega$ is a disk with radius $a$ and centered at the origin $\mathbf{O}$. In this case, the singlelayer operator $L$ can be diagonalized in the Fourier basis associated with the circular cylinder and results in the diagonal infinite matrix $\widetilde{\mathbb{L}}$ representation with coefficients $\widetilde{\mathbb{L}}_{m n}$ given by [21]

$$
\forall m, n \in \mathbb{Z}, \quad \widetilde{\mathbb{L}}_{m n}=\delta_{m n} \frac{i \pi a}{2} J_{m}(k a) H_{m}^{(1)}(k a) .
$$

Symbol $\delta_{m n}$ is the Krönecker's delta function, equal to 1 if $m=n$ and 0 otherwise. Functions $J_{m}$ and $H_{m}^{(1)}$ are the Bessel and first-kind Hankel functions of order $m$. Thus, for one disk, the singlelayer operator is diagonal with coefficients: $\mu_{m}=\widetilde{\mathbb{L}}_{m m}$. Furthermore, the operator $L$ is singular if one of its eigenvalues $\mu_{m}$ is equal to zero, which means that $J_{m}(k a)=0$, for a certain value of $m$ for $k a$ fixed (let us recall that $H_{m}^{(1)}(x) \neq 0$ for any $x>0$ ). This is not the case here since we assume that $k \notin F_{D}(\Omega)=\left\{k / \exists m \in \mathbb{N} / J_{m}(k a)=0\right\}$. For the finite dimensional approximation, we keep $2 N+1$ modes such that the indices $m$ satisfy $-N \leq m \leq N$. We denote by $\mathbb{L}=\left(\mathbb{L}_{m n}\right)_{-N \leq m, n \leq N}$ the diagonal matrix of size $(2 N+1) \times(2 N+1)$ which approximates the single-layer operator. Its coefficients are

$$
\forall-N \leq m, n \leq N, \quad \mathbb{L}_{m n}=\widetilde{\mathbb{L}}_{m n}=\delta_{m n} \frac{i \pi a}{2} J_{m}(k a) H_{m}^{(1)}(k a) .
$$

The eigenvalues $\mu_{m}, m=-N, \ldots, N$, of the diagonal matrix $\mathbb{L}$ are explictly given by

$$
\mu_{m}=\mathbb{L}_{m m}=\frac{i \pi a}{2} J_{m}(k a) H_{m}^{(1)}(k a), \quad m=-N, \ldots, N .
$$

Let us remark that, following $[1,(9.1 .5)]$, we have

$$
\forall m \in \mathbb{Z}, \forall x \neq 0, \quad H_{-m}^{(1)}(x)=(-1)^{m} H_{m}^{(1)}(x), \quad J_{-m}(x)=(-1)^{m} J_{m}(x) .
$$

These properties imply that the eigenvalues $\mu_{m}$ of $\mathbb{L}$ are double: $\mu_{m}=\mu_{-m}, m=-N, \ldots, N, m \neq 0$, the eigenvalue $\mu_{0}$ being single.

Since the eigenvalues are given by special functions, the dependence in terms of dimensionless wave number $k a$ and truncation parameter $N$ is not easy to analyze. To get simpler expressions we consider low frequency expansions of the eigenvalues. Let us remark that the medium/high frequency analysis is also possible $[5,6]$ but its extension to multiple scattering is more tricky. 
For this reason, we restrict ourselves to the low frequency regime. For $k a \rightarrow 0$, the asymptotic expansions of $J_{m}$ and $H_{m}^{(1)}$ give (see relations (9.1.12)-(9.1.13) [1])

$$
\mu_{m} \simeq \begin{cases}-a\left[\ln \left(\frac{k a}{2}\right)+\gamma\right]+i \frac{\pi a}{2}+O\left((k a)^{2} \ln (k a)\right) & \text { for } m=0 \\ \frac{a}{2|m|}+O\left((k a)^{2}\right) & \text { for } m \neq 0\end{cases}
$$

where $\gamma=0.577 \ldots$ is the Euler's constant. To illustrate and validate this approximation, we report on Figure 2 the real and imaginary parts of the exact (3) and approximate (5) eigenvalues by truncating the approximation to $N=20$, for $k a=0.2(a=1$ here). We observe that the approximation provides close values to the expected ones. Moreover, we remark that the eigenvalue associated with the mode $m=0$ is the only one to get a significant imaginary part since the zero order mode is the only propagating one at low frequency (this is not the case for a higher frequency). For larger $k a$, the considered approximations introduce more errors. Furthermore, we see that the modulii of the eigenvalues of $\mathbb{L}$ tend towards zero when $m$ is large enough. These eigenvalues, corresponding to evanescent modes, have zero as limit since $L$ is a (pseudodifferential) operator of order -1 , having therefore eigenvalues associated to high order spatial modes going to zero.

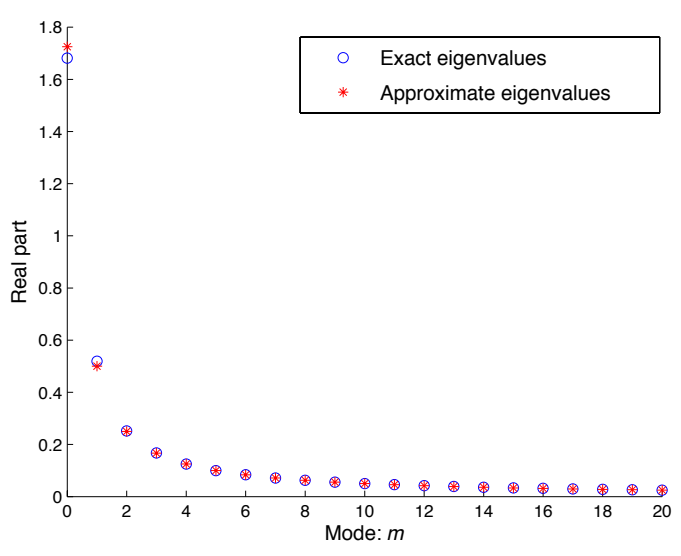

(a) Real parts

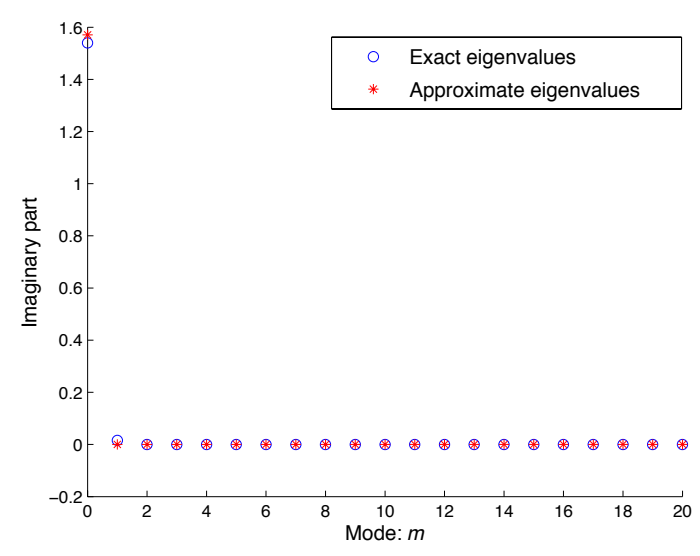

(b) Imaginary parts

Figure 1: Comparison between the exact (3) and approximate (5) eigenvalues of $L$, for $k a=0.2$ and $N=20$.

The condition number cond $(\mathbb{L})$ of $\mathbb{L}$ is defined by: $\operatorname{cond}(\mathbb{L})=\|\mathbb{L}\|\left\|\mathbb{L}^{-1}\right\|$, for a matrix norm $\|\cdot\|$ associated with a vectorial norm $\|\cdot\|$ on $\mathbb{C}^{2 N+1}$, that is

$$
\|\mathbb{L}\|=\sup _{\mathbf{X} \in \mathbb{C}^{2 N+1} \backslash\{0\}} \frac{\|\mathbb{L} \mathbf{X}\|}{\|\mathbf{X}\|} .
$$

If the 2-norm $\|\cdot\|_{2}$ is defined by $\|\mathbf{X}\|_{2}=\sqrt{\sum_{m=-N}^{N}\left|X_{m}\right|^{2}}$, for any vector $\mathbf{X}=\left(X_{-N}, \ldots, X_{N}\right)^{T} \in$ $\mathbb{C}^{2 N+1}$, we have $\operatorname{cond}_{2}(\mathbb{L})=\|\mathbb{L}\|_{2}\left\|\mathbb{L}^{-1}\right\|_{2}$. Since $\mathbb{L}$ is diagonal, it is normal and $\operatorname{cond}_{2}(\mathbb{L})=\frac{\left|\mu_{\max }\right|}{\left|\mu_{\min }\right|}$, where $\left|\mu_{\max }\right|=\max _{0 \leq m \leq N}\left|\mu_{m}\right|$ and $\left|\mu_{\min }\right|=\min _{0 \leq m \leq N}\left|\mu_{m}\right|$. From the previous low frequency 
analysis (5), the eigenvalue with maximal modulus is obtained for $m=0$ (propagating mode) and the one with minimal modulus corresponds to $m=N$ (evanescent mode). Finally, a low frequency approximation $(k a \ll 1)$ of the condition number is

$$
\operatorname{cond}_{2}(\mathbb{L}) \simeq\left\{a^{2}\left[\ln \left(\frac{k a}{2}\right)+\gamma\right]^{2}+\left(\frac{\pi a}{2}\right)^{2}\right\}^{1 / 2} \frac{2 N}{a},
$$

for a circular cylinder of radius $a$ with truncation Fourier parameter $N$.

Remark. A similar analysis can be considered for the sphere to understand the three-dimensional situation where spherical harmonics can be used to diagonalize the integral operators (see [6] for the high-frequency single scattering case).

\subsection{Link with the boundary element approximation and formal extensions to other objects}

Let us now introduce the boundary element approximation of (1), assuming that the boundary $\Gamma$ is sufficiently smooth. Let $\Gamma_{h}=\cup_{j=1}^{N_{h}} K_{j}$ be a polygonal approximation of $\Gamma$ by using $N_{h}$ segments $K_{j}$, $1 \leq j \leq N_{h}$. We designate by $h_{j}$ the length of $K_{j}$ and by $h$ the maximal length: $h=\max _{1 \leq j \leq N_{h}} h_{j}$. We choose the boundary element subspace $V_{h}$ of $L^{2}\left(\Gamma_{h}\right)$ with continuous piecewise linear functions over $\Gamma_{h}$

$$
V_{h}:=\left\{\rho_{h} \in \mathcal{C}^{0}\left(\Gamma_{h}\right) /\left.\rho_{h}\right|_{K_{j}} \in \mathbb{P}_{1}, 1 \leq j \leq N_{h}\right\}
$$

We define by $\left[L_{h}\right] \in \mathcal{M}_{N_{h}, N_{h}}(\mathbb{C})$ the matrix associated with the discretization of the single-layer operator and by $\left[M_{h}\right] \in \mathcal{M}_{N_{h}, N_{h}}(\mathbb{C})$ the mass matrix for $V_{h}$.

\subsubsection{The circular cylinder}

We now relate the spectral Fourier approximation to the boundary element method. Let us assume that the mesh is uniform with step $h$. The number of degrees of freedom $N_{E}$ is then: $N_{E}=$ $N_{h} \simeq \frac{2 \pi a}{h}$. The spectral method requires $2 N+1$ modes. Let $\mu_{\min }^{h}$ and $\mu_{\max }^{h}$ be the eigenvalues of $\left[M_{h}\right]^{-1}\left[L_{h}\right]$ with smallest and largest modulus, respectively. Formally replacing $N$ by $\pi a h^{-1}-1 / 2$ in the estimates $\mu_{\min }$ and $\mu_{\max }$, we get

$$
\mu_{\min }(a, h) \simeq \frac{a}{2 \pi a h^{-1}-1}, \quad \mu_{\max }(k, a) \simeq-a\left[\ln \left(\frac{k a}{2}\right)+\gamma\right]+i\left(\frac{\pi a}{2}\right)
$$

We then obtain the following estimate of the spectral condition number (6)

$$
\operatorname{cond}\left(k, a, \Gamma_{h}\right):=\operatorname{cond}_{2}\left(\left[M_{h}\right]^{-1}\left[L_{h}\right]\right) \simeq \operatorname{cond}_{a p p}(k, a, h),
$$

with

$$
\operatorname{cond}_{\text {app }}(k, a, h)=\left\{a^{2}\left[\ln \left(\frac{k a}{2}\right)+\gamma\right]^{2}+\left(\frac{\pi a}{2}\right)^{2}\right\}^{1 / 2} \frac{2 \pi a h^{-1}-1}{a},
$$

when $k a$ tends towards zero.

To validate (9), we set $a=1$ and consider the following test cases. We compare on Figures 2 (a) and 2(b) the numerical condition number $\operatorname{cond}\left(k, a, \Gamma_{h}\right)$ and its estimate $\operatorname{cond}_{a p p}(k, a, h)$ for a uniform mesh. In the first case, we let $k a$ varies for $N_{h}=100$. In the second case, the number of 
points $N_{h}$ varies for $k a=0.1$. In both situations, we see that our estimate is accurate. Moreover, we clearly observe that, when $k a$ is large enough, we lose some accuracy since the low frequency approximation is no longer valid. We consider now on Figures 2(c) and 2(d) a non uniform mesh. To this end, we generate the sequence of segments $K_{j}=\left[\mathbf{x}_{j-1}, \mathbf{x}_{j}\right], 1 \leq j \leq N_{h}$, with

$$
\mathbf{x}_{j-1}=a\left(\cos \left(\theta_{j-1}\right), \sin \left(\theta_{j-1}\right)\right), \quad \theta_{j-1}=\frac{2 \pi}{N_{h}}\left(j-1+\frac{10 \varepsilon_{j-1}}{N_{h}}\right),
$$

where $\varepsilon_{j-1}$ is a random variable of uniform law on $[0 ; 1]$ and $\mathbf{x}_{N_{h}}=\mathbf{x}_{0}$. The computation of $\operatorname{cond}\left(k, a, \Gamma_{h}\right)$ is again realized numerically or approximated via formula (10). Here, the choice of $h$ is more delicate. Indeed, the dependence of the condition number cond $\left(k, a, \Gamma_{h}\right)$ according to the geometry $\Gamma_{h}$ is a priori global and affects $\mu_{\min }(a, h)$. In the case of a uniform discretization, we may consider an estimate of $\mu_{\min }(a, \cdot)$ which is only local by taking $\operatorname{cond}_{a p p}\left(k, a, h_{\text {min }}\right)$ or $\operatorname{cond}_{a p p}\left(k, a, h_{\text {max }}\right)$, with $h_{\text {min }}=\min _{1 \leq j \leq N_{h}} h_{j}$ and $h_{\max }=\max _{1 \leq j \leq N_{h}} h_{j}$, or global by considering $\operatorname{cond}_{a p p}\left(k, a, h_{\text {eqv }}\right)$, with $h_{\text {eqv }}=N_{h}^{-1} \sum_{j=1}^{N_{h}} h_{j}$. This last choice corresponds to a calculation of the conditioning based on an equivalent uniform discretization for an average step $h_{\text {eqv }}$. For a uniform discretization, the definitions coincide. We finally remark that the choice of $h_{\text {eqv }}$ leads to the most accurate estimates.

\subsubsection{Estimates to other geometries}

We now consider two other geometries a) an elliptical scatterer of semi-axes $a_{x_{1}}$ along $\overrightarrow{\mathbf{O} x_{1}}$ and $a_{x_{2}}$ along $\overrightarrow{\mathbf{O} x_{2}}$, and b) a rectangular cylinder with side-lengths $2 a_{x_{1}}$ and $2 a_{x_{2}}$ along $\overrightarrow{\mathbf{O} x_{1}}$ and $\overrightarrow{\mathbf{O} x_{2}}$. These two objects are centered at the origin.

For the first case, let us recall that the approximation of the eigenvalues with smallest and largest modulus for $\mathbb{P}_{1}$ boundary element are estimated respectively by

$$
\mu_{\min }(a, h) \simeq \frac{a}{2 \pi a h^{-1}-1}, \quad \mu_{\max }(k, a) \simeq a\left[\ln \left(\frac{k a}{2}\right)+\gamma\right]+i \frac{\pi a}{2} .
$$

Then we propose to answer the following two questions: how to handle an equivalent radius, denoted by $a_{\text {eqv }}$, for an ellipse, and, since the mesh is non uniform, which mesh step $h$ can be chosen. For the ellipse, we propose the three following equivalent radii for the approximation of the eigenvalue with largest modulus

$$
a_{\text {eqv }}^{1}=\frac{a_{x_{1}}+a_{x_{2}}}{2}, \quad a_{\text {eqv }}^{2}=\frac{2 a_{x_{1}} a_{x_{2}}}{a_{x_{1}}+a_{x_{2}}}, \quad a_{\text {eqv }}^{3}=\frac{\sqrt{a_{x_{1}}^{2}+a_{x_{2}}^{2}}}{\sqrt{2}} .
$$

The first approximation consists in considering an equivalent disk with mean radius based on $a_{x_{1}}$ and $a_{x_{2}}$ and the second one with a curvature given by the mean of the curvatures $1 / a_{x_{1}}$ and $1 / a_{x_{2}}$. The last radius is based on taking the point $\left(a_{x_{1}}, a_{x_{2}}\right) / \sqrt{2}$ of the ellipse and next choosing the radius of the circle centered at the origin and passing by this point. Concerning the discretization, we propose three possibilities related to $h_{\min }, h_{\max }$ and $h_{\text {eqv }}$, for each value $a_{\text {eqv }}^{j}, j=1,2,3$. Let us fix the following configuration: $a_{x_{1}}=1, a_{x_{2}}=0.25$ and $N_{h}=60$. We report on Figures $3(\mathrm{a}), 3(\mathrm{~b})$ and 3(c) respectively the smallest and largest eigenvalues (with respect to their modulii) and the condition number vs. $k$. We remark that the eigenvalue with largest modulus is well approximated by $a_{\text {eqv }}^{3}$ and the smallest one is not sensitive to this parameter (as expected). The 


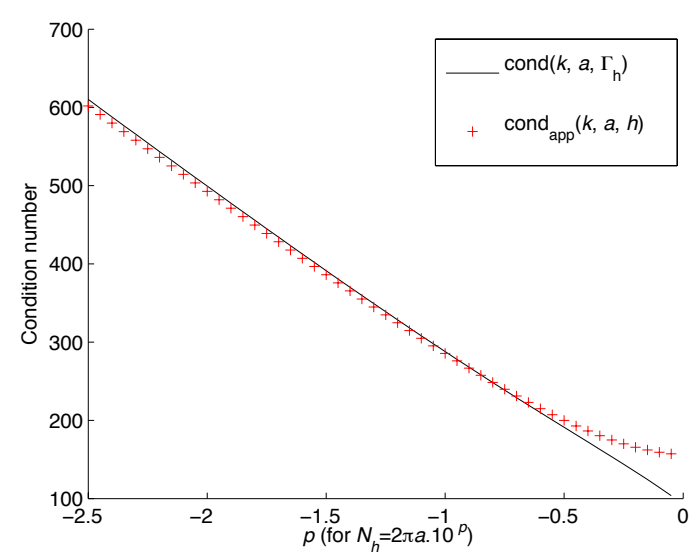

(a) Condition number vs. $k a, N_{h}=100$ (uniform discretization)

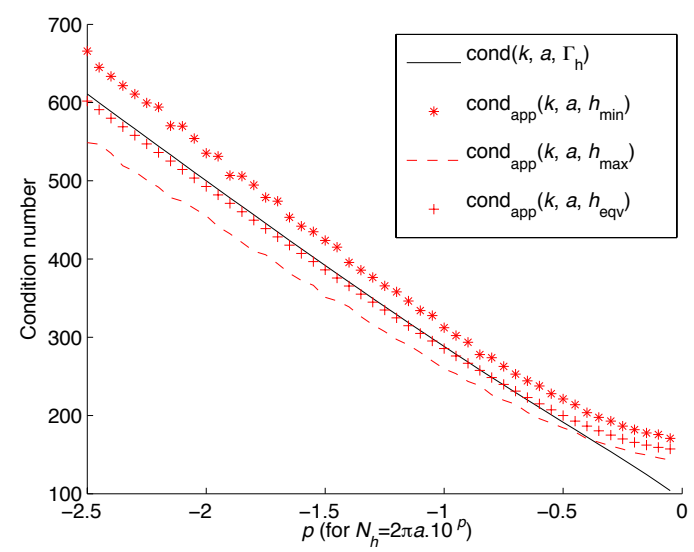

(c) Condition number vs. $k a, N_{h}=100$ (non uniform discretization)

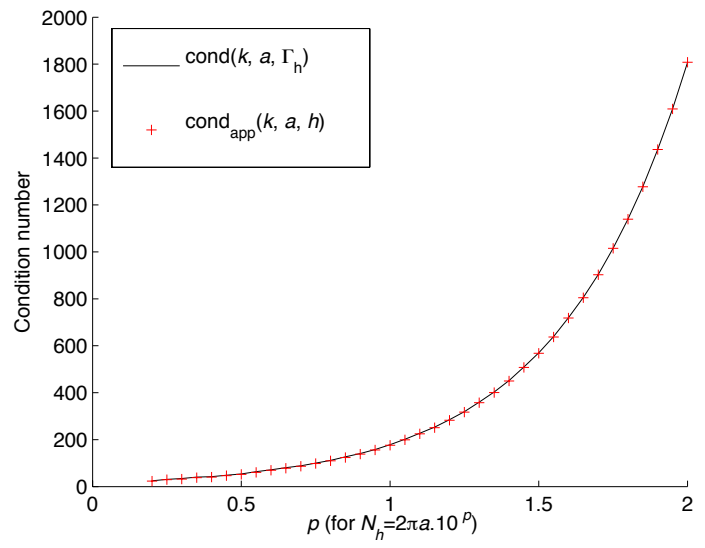

(b) Condition number vs. $N_{h}, k a=0.1$ (uniform discretization)

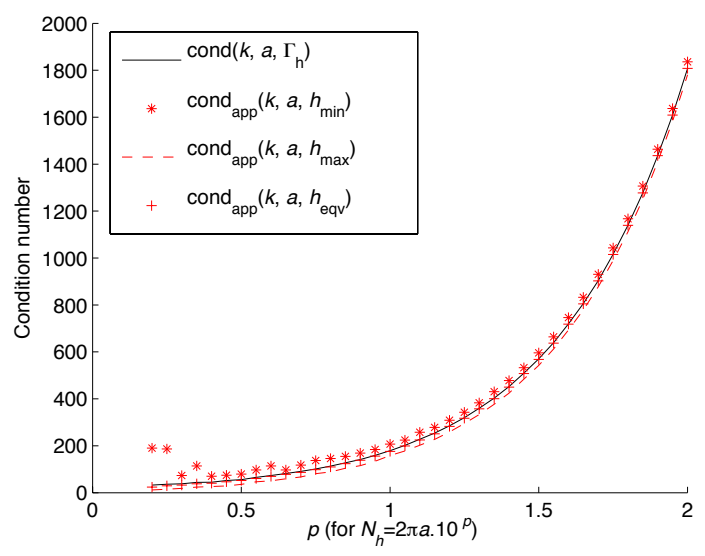

(d) Condition number vs. $N_{h}, k a=0.1$ (non uniform discretization)

Figure 2: Validation of the approximation formulae for the condition number of the single-layer potential in the circular cylinder case (uniform and non uniform meshes).

choice of the equivalent discretization step is however crucial and the best approximation occurs for $h_{\text {min }}$. Further test cases have been realized and always lead to the same conclusion, that is the approximations of the condition number is satisfactory. We compare on Figure 4 the different estimates according to the ratio between the different axes. Let us fix $N_{h}=60, k=0.2$ and $a_{x_{1}}=1$, and let $a_{x_{2}}$ varies between 0.05 and 1 . We can again see that using $a_{\text {eqv }}^{3}$ is the best choice. Furthermore, $\mu_{\min }^{h}$ is obtained for $h_{\min }$. We retain this choice of parameters for the estimates of both the eigenvalues and condition number of the matrix $\left[M_{h}\right]^{-1}\left[L_{h}\right]$.

For the rectangular cylinder with sidelengths $a_{x_{1}}$ and $a_{x_{2}}$, we choose the following approximation

$$
a_{\mathrm{eqv}}^{4}=\frac{(1+\sqrt{2})}{2} \frac{\sqrt{a_{x_{1}}^{2}+a_{x_{2}}^{2}}}{\sqrt{2}} .
$$




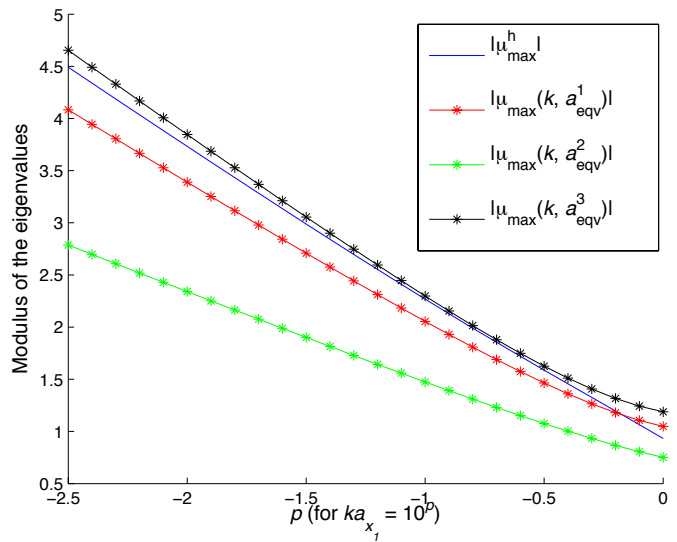

(a) Numerical and approximate eigenvalues with largest modulus.

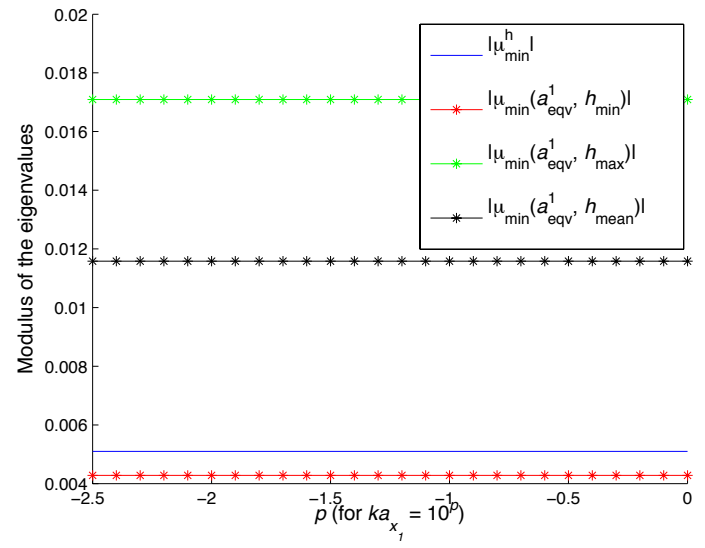

(b) Numerical and approximate eigenvalues with smallest modulus.

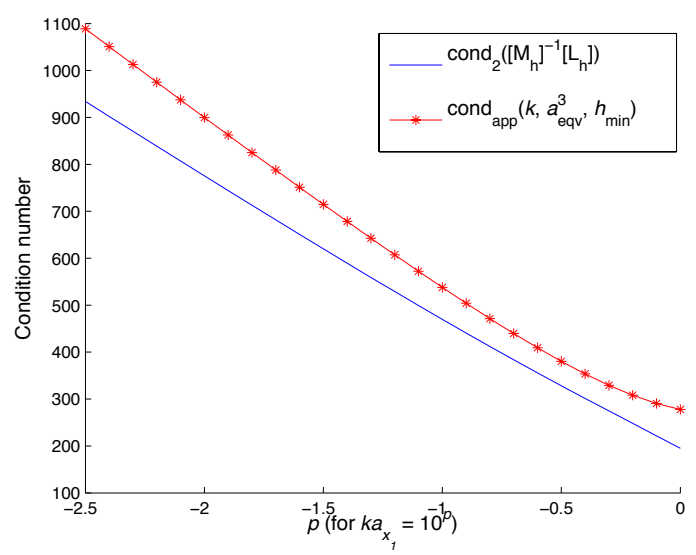

(c) Numerical and approximate condition numbers.

Figure 3: Validation of the approximation formulae of the condition number for the single-layer potential and the ellipse vs. $k a_{x_{1}}\left(a_{x_{1}}=1, a_{x_{2}}=0.25\right.$ and $\left.N_{h}=60\right)$ (non uniform mesh).

To get this equivalent radius, we consider two ellipses: one with semi-axes $a_{x_{1}}$ and $a_{x_{2}}$ and another one with semi-axes $\sqrt{2} a_{x_{2}}$ and $\sqrt{2} a_{x_{2}}$ (along the abscissa and ordinates). In particular, the corners of the rectangle own to this last ellipse while the middle points of the faces are on the first one. Next, we consider the ellipse with semi-axes equal to the average of the semi-axes of the two ellipses, that is with semi-axes $(1+\sqrt{2}) a_{x_{1}} / 2$ and $(1+\sqrt{2}) a_{x_{2}} / 2$. Finally, the disc with the equivalent radius $a_{\text {eqv }}^{3}$ for the previous ellipse is considered leading to (13). We report on Figures 5(a) to 5(c) the approximation of the largest and smallest approximate eigenvalues and the corresponding condition number. We take $a_{x_{1}}=1$ and $a_{x_{2}}=0.25$ and discretize each side by using 50 points, for a total number of $N_{h}=200$ points. As we can see, the approximation based on $a_{\text {eqv }}^{4}$ is accurate and the choice of the minimal parameter is very satisfactory.

As a conclusion, this first study provides some explicit estimates of the eigenvalues with minimal and maximal modulii as well as condition number estimates of the single-layer potential in the low 

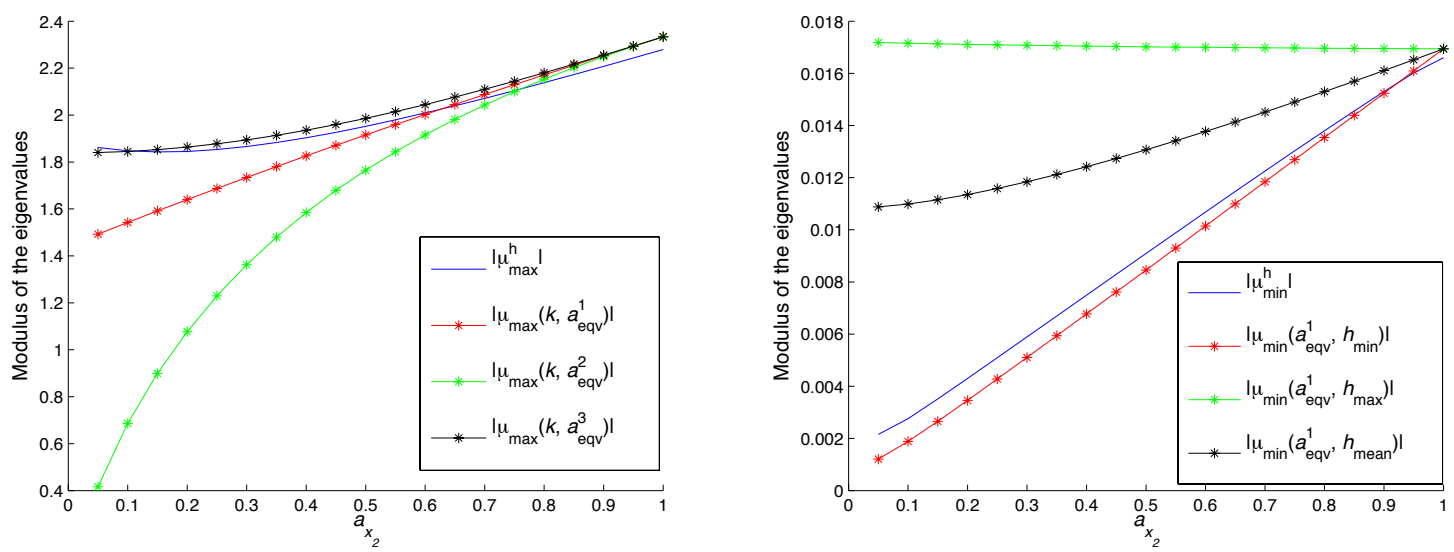

(a) Numerical and approximate eigenvalues with largest (b) Numerical and approximate eigenvalues with smallmodulus. est modulus.

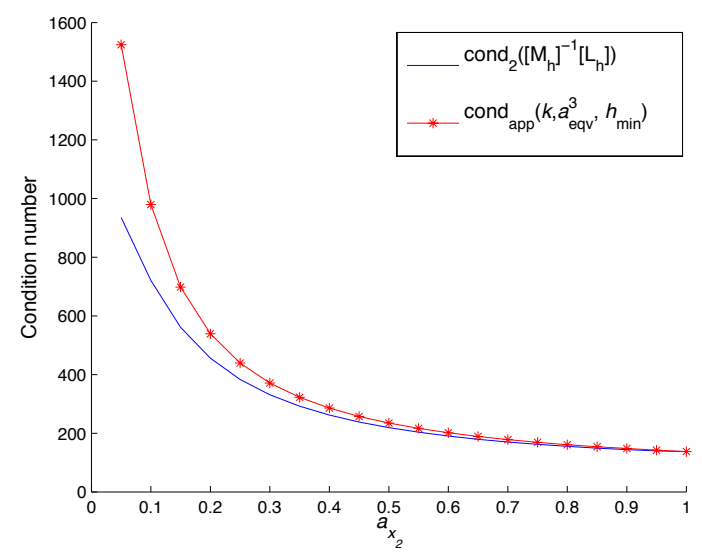

(c) Numerical and approximate condition numbers.

Figure 4: Validation of the approximation formulae of the condition number for the single-layer potential and the ellipse (non uniform mesh), for $N_{h}=60, k=0.2, a_{x_{1}}=1$ and $a_{x_{2}}$ varying between 0.05 and 1 .

frequency regime and for the circular, the elliptical and rectangular cylinders.

\section{Spectral estimates for multiple scattering in dilute media}

\subsection{The single-layer operator for multiple scattering by disks}

We assume now that $\Omega$ is the union of $M$ strictly disjoint (no sticky case) circular cylinders $\Omega_{p}$, $p=1, \ldots, M$. The boundary $\Gamma_{p}$ of $\Omega_{p}$ is a circle with center $\mathbf{O}_{p}$ and radius $a_{p}$. The explicit 


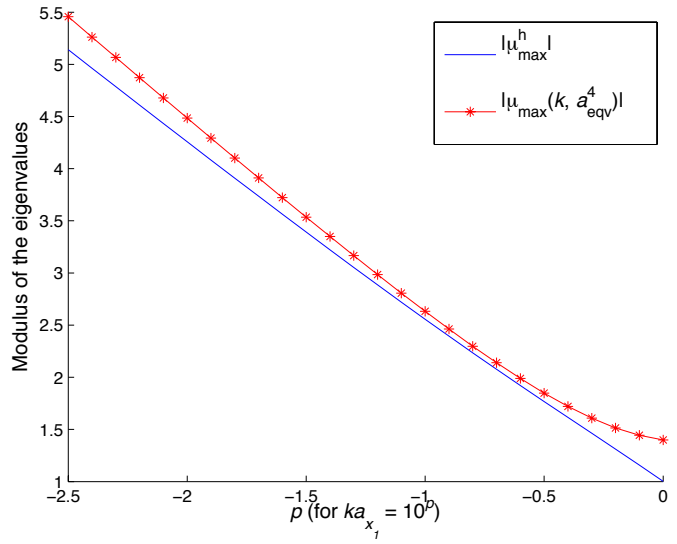

(a) Numerical and approximate eigenvalues with largest modulus.

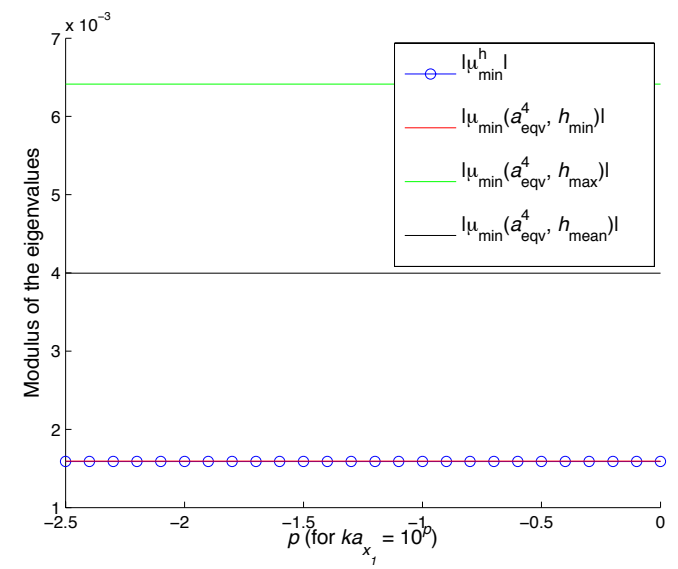

(b) Numerical and approximate eigenvalues with smallest modulus.

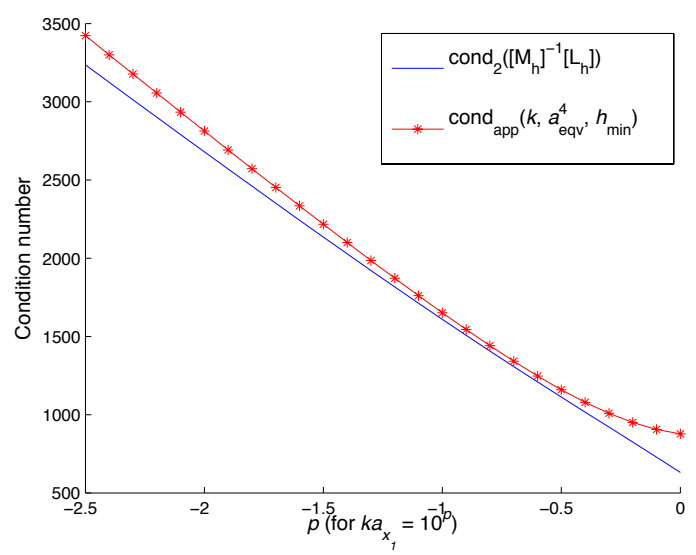

(c) Numerical and approximate condition numbers.

Figure 5: Validation of the approximation formulae for the eigenvalues $\mu_{\min }^{h}$ and $\mu_{\max }^{h}$ and the condition number for the single-layer potential and a rectangular cylinder with half side lengths $a_{x_{1}}=1$ and $a_{x_{2}}=0.25$, vs. $k a_{x_{1}}\left(N_{h}=200\right.$ segments, 50 for each side).

expression of the single-layer potential in the case of several disks has been obtained e.g. in [26] as

$$
\widetilde{\mathbb{L}}:=\left(\begin{array}{cccc}
\widetilde{\mathbb{L}}^{1,1} & \widetilde{\mathbb{L}}^{1,2} & \ldots & \widetilde{\mathbb{L}}^{1, M} \\
\widetilde{\mathbb{L}}^{2,1} & \widetilde{\mathbb{L}}^{2,2} & \ldots & \widetilde{\mathbb{L}}^{2, M} \\
\vdots & \vdots & \ddots & \vdots \\
\widetilde{\mathbb{L}}^{M, 1} & \widetilde{\mathbb{L}}^{M, 2} & \ldots & \widetilde{\mathbb{L}}^{M, M}
\end{array}\right) .
$$


The coefficients of the single-layer potential, for two objects $p$ and $q$, with $p, q=1, \ldots, M$, for two Fourier modes $m, n \in \mathbb{Z}$, are given by the expressions

$$
\widetilde{\mathbb{L}}_{m, n}^{p, q}= \begin{cases}\frac{i \pi a_{p}}{2} J_{m}\left(k a_{p}\right) H_{m}^{(1)}\left(k a_{p}\right) \delta_{m n} & \text { if } p=q, \\ \frac{i \pi \sqrt{a_{p} a_{q}}}{2} J_{m}\left(k a_{p}\right) S_{n m}\left(b_{p q}\right) J_{n}\left(k a_{q}\right) & \text { otherwise. }\end{cases}
$$

The quantity $S_{n m}\left(b_{p q}\right)$ is given by: $S_{n m}\left(b_{p q}\right)=H_{n-m}^{(1)}\left(k b_{p q}\right) e^{i(n-m) \alpha_{p q}}$, for $p, q=1, \ldots, M, p \neq q$ and $m, n \in \mathbb{Z}$. Here, the different quantities are defined by

$$
\mathbf{b}_{p q}=\mathbf{O}_{q} \mathbf{O}_{p}, \quad b_{p q}=\left\|\mathbf{b}_{p q}\right\|, \quad \alpha_{p q}=\operatorname{Angle}\left(\overrightarrow{\mathbf{O} x_{1}}, \mathbf{O}_{q} \mathbf{O}_{p}\right) .
$$

Let us now consider a finite dimensional projection. To this end, we truncate system (14) by keeping, for each Fourier series (associated to an object $p=1, \ldots, M$ ), $2 N_{p}+1$ modes such that: $-N_{p} \leq m \leq N_{p}$. The resulting truncated matrix is then

$$
\mathbb{L}:=\left(\begin{array}{cccc}
\mathbb{L}^{1,1} & \mathbb{L}^{1,2} & \ldots & \mathbb{L}^{1, M} \\
\mathbb{L}^{2,1} & \mathbb{L}^{2,2} & \cdots & \mathbb{L}^{2,1} \\
\vdots & \vdots & \ddots & \vdots \\
\mathbb{L}^{M, 1} & \mathbb{L}^{M, 2} & \cdots & \mathbb{L}^{M, M}
\end{array}\right)
$$

where each block $\mathbb{L}^{p, q}$, of size $\left(2 N_{p}+1\right) \times\left(2 N_{q}+1\right)$, for $p, q=1, \ldots, M$, of matrix $\mathbb{L}$ has coefficients (15): $\mathbb{L}_{m, n}^{p, q}=\widetilde{\mathbb{L}}_{m, n}^{p, q}$, for $-N_{p} \leq m \leq N_{p}$ and $-N_{q} \leq n \leq N_{q}$.

\subsection{Asymptotic spectral analysis for a collection of disks}

To simplify the analysis, we assume here that: $\forall p=1, \ldots, M, a_{p}=a$. We again consider the low frequency regime $k a \rightarrow 0$. Unlike the single-scattering case, we do not have access directly to the eigenvalues of $\mathbb{L}$ for multiple scattering configurations since the matrix is not diagonal. Furthermore, new parameters are involved in the analysis, essentially $k b_{p q}$, where $b_{p q}$ is the distance between the centers of $\Omega_{p}$ and $\Omega_{q}$. We propose to analyze the effect of these parameters on the condition number of the single-layer operator. Partial results can be obtained for some regimes, the general case being out of reach. We analyze here the case of far obstacles (called dilute media in our paper) (see [7] for close obstacles). In [26], we proved that an approach based on Gershgorin discs does not provide precise estimates. For this reason, we propose an alternative approach.

Let us first consider the diagonal block $\mathbb{L}^{p, p}$, for $p=1, \ldots, M$ which is a diagonal matrix. When $k a \rightarrow 0$, its coefficients $\mathbb{L}_{m, m}^{p, p}$, for $-N \leq m \leq N$ satisfy [26]

$$
\mathbb{L}_{m, m}^{p, p}= \begin{cases}\widehat{\mathbb{L}}_{0}+O\left((k a)^{2} \ln (k a)\right) & \text { if } m=0, \\ \widehat{\mathbb{L}}_{m}+O\left((k a)^{2}\right) & \text { otherwise }\end{cases}
$$

with

$$
\widehat{\mathbb{L}}_{m}= \begin{cases}-a\left[\ln \left(\frac{k a}{2}\right)+\gamma\right]+\frac{i \pi a}{2} & \text { if } m=0 \\ \frac{a}{2|m|} & \text { otherwise. }\end{cases}
$$


We build the diagonal submatrix $\left(\mathbb{L}^{0}\right)^{p, p}$ which only contains the dominating terms of the asymptotic expansion obtained in (16). More precisely, the $(2 N+1) \times(2 N+1)$ matrix $\left(\mathbb{L}^{0}\right)^{p, p}$ is defined by

$$
\left(\mathbb{L}^{0}\right)^{p, p}=\left(\begin{array}{ccccc}
\widehat{\mathbb{L}}_{N} & 0 & 0 & \cdots & 0 \\
0 & \ddots & \ddots & & 0 \\
\vdots & \ddots & \widehat{\mathbb{L}}_{0} & \ddots & \vdots \\
0 & & \ddots & \ddots & 0 \\
0 & \cdots & 0 & 0 & \widehat{\mathbb{L}}_{N}
\end{array}\right)
$$

Relation (16) implies that, when $k a \rightarrow 0$, the following relation is fulfilled, for $p=1, \ldots, M$

$$
\mathbb{L}^{p, p}=\left(\mathbb{L}^{0}\right)^{p, p}+O\left((k a)^{2} \ln (k a)\right) .
$$

In other words, each submatrix $\left(\mathbb{L}^{0}\right)^{p, p}$ is an approximation of the diagonal block $\mathbb{L}^{p, p}$ of matrix $\mathbb{L}$. From relation $(18)$, we see that the coefficients of $\left(\mathbb{L}^{0}\right)^{p, p}$ do not depend on the index $p$ (since $a_{p}=a$, for all $\left.p=1, \ldots, M\right)$. Hence, for $1 \leq p, q \leq M$, we have the equality $\left(\mathbb{L}^{0}\right)^{p, p}=\left(\mathbb{L}^{0}\right)^{q, q}$.

We now proceed in a similar way for the off-diagonal block $\mathbb{L}^{p, q}$, with $1 \leq p, q \leq M$ and $p \neq q$. When $k a \rightarrow 0$ and $k b_{p q} \rightarrow+\infty$, we have [26]

$$
\mathbb{L}_{m, n}^{p, q}= \begin{cases}i a \sqrt{\frac{\pi}{2}} \frac{e^{i\left(k b_{p q}-\pi / 4\right)}}{\sqrt{k b_{p q}}}+O\left(\frac{1}{k b_{p q}}\right)+O\left(\frac{(k a)^{2}}{\sqrt{k b_{p q}}}\right) & \text { if }(m, n)=(0,0), \\ O\left(\frac{(k a)^{|m|+|n|}}{\sqrt{k b_{p q}}}\right) & \text { if }(m, n) \neq(0,0) .\end{cases}
$$

Hence, all the coefficients $\mathbb{L}_{m, n}^{p, q}$ of $\mathbb{L}^{p, q}$ decay (at least) like

$$
O\left(\frac{k a}{\sqrt{k b_{p q}}}\right)
$$

except the term of indices $(m, n)=(0,0)$, which decays like

$$
O\left(\frac{1}{\sqrt{k b_{p q}}}\right) \text {. }
$$

Let $\left(\mathbb{L}^{0}\right)^{p, q}$ be the submatrix of size $(2 N+1) \times(2 N+1)$ defined by

$$
\left(\mathbb{L}^{0}\right)^{p, q}=\left(\begin{array}{ccccc}
0 & 0 & 0 & \ldots & 0 \\
0 & \ddots & \ddots & & 0 \\
\vdots & \ddots & \left(\mathbb{L}^{0}\right)_{0,0}^{p, q} & \ddots & \vdots \\
0 & & \ddots & \ddots & 0 \\
0 & \ldots & 0 & 0 & 0
\end{array}\right) \quad \text { with } \quad\left(\mathbb{L}^{0}\right)_{0,0}^{p, q}=i a \sqrt{\frac{\pi}{2}} \frac{e^{i\left(k b_{p q}-\pi / 4\right)}}{\sqrt{k b_{p q}}}
$$

Let us introduce the parameter: $b=\min _{p, q=1, \ldots, M} b_{p q}$. By using (20), when $k a \rightarrow 0$ and $k b \rightarrow+\infty$, the following relation holds, for $p \neq q=1, \ldots, M, p \neq q$

$$
\mathbb{L}^{p, q}=\left(\mathbb{L}^{0}\right)^{p, q}+O\left(\frac{k a}{\sqrt{k b_{p q}}}\right)+O\left(\frac{1}{k b_{p q}}\right) .
$$


Hence, each block $\left(\mathbb{L}^{0}\right)^{p, q}$ is constituted of an approximation of the block $\mathbb{L}^{p, q}$ of $\mathbb{L}$. Let us introduce the block matrix $\mathbb{L}^{0}$ which contains each submatrix $\left(\mathbb{L}^{0}\right)^{p, q}$

$$
\mathbb{L}^{0}=\left(\begin{array}{cccc}
\left(\mathbb{L}^{0}\right)^{1,1} & \left(\mathbb{L}^{0}\right)^{1,2} & \ldots & \left(\mathbb{L}^{0}\right)^{1, M} \\
\left(\mathbb{L}^{0}\right)^{2,1} & \left(\mathbb{L}^{0}\right)^{2,2} & \ldots & \left(\mathbb{L}^{0}\right)^{2, M} \\
\vdots & \vdots & \ddots & \vdots \\
\left(\mathbb{L}^{0}\right)^{M, 1} & \left(\mathbb{L}^{0}\right)^{M, 2} & \ldots & \left(\mathbb{L}^{0}\right)^{M, M}
\end{array}\right)
$$

Let us summarize these results in the next Lemma.

Lemma 1. When $k a \longrightarrow 0$ and $k b \longrightarrow+\infty$, we have

$$
\mathbb{L}=\mathbb{L}^{0}+O\left((k a)^{2} \ln (k a)\right)+O\left(\frac{k a}{\sqrt{k b}}\right)+O\left(\frac{1}{k b}\right) .
$$

From now on, we respectively denote by $\left(\mu_{m}^{p}\right)_{1 \leq p \leq M,-N \leq m \leq N}$ and $\left(\left(\mu^{0}\right)_{m}^{p}\right)_{1 \leq p \leq M,-N \leq m \leq N}$ the eigenvalues of $\mathbb{L}$ and $\mathbb{L}^{0}$. For $p=1, \ldots, M$ and $m=-N, \ldots, N$, we assume that: $\mu_{m}^{p} \simeq\left(\mu^{0}\right)_{m}^{p}$, which is coherent with (23). To motivate our approach and to understand how the eigenvalues of $\mathbb{L}^{0}$ are distributed, we compare on Figure 6 the eigenvalues of $\mathbb{L}$ and $\mathbb{L}^{0}$, for 50 unitary disks randomly distributed in $[0,1000]^{2}$, with $k a=0.1, k b \geq 10$ and $N=4$. The eigenvalues are computed numerically with the help of the eig Matlab function. Globally, the approximation of the eigenvalues $\left(\mu_{m}^{p}\right)_{p=1, \ldots, M, m=-N, \ldots, N}$ of $\mathbb{L}$ by the eigenvalues $\left(\left(\mu^{0}\right)_{m}^{p}\right)_{p=1, \ldots, M, m=-N, \ldots, N}$ of $\mathbb{L}^{0}$ are satisfactory.

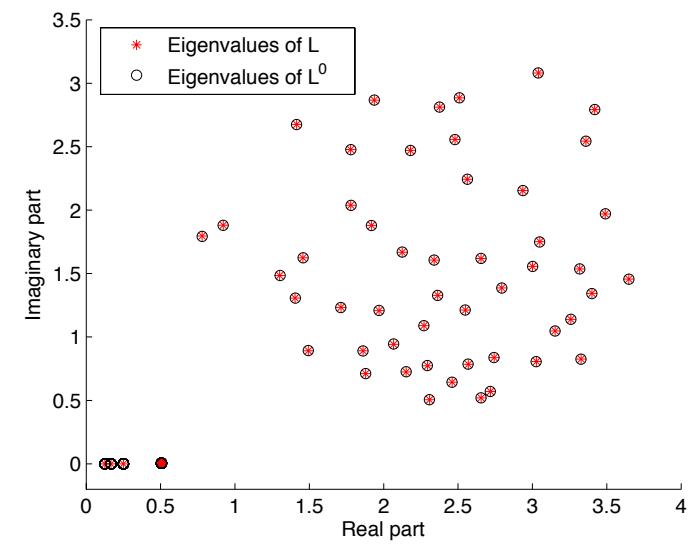

(a) All the eigenvalues

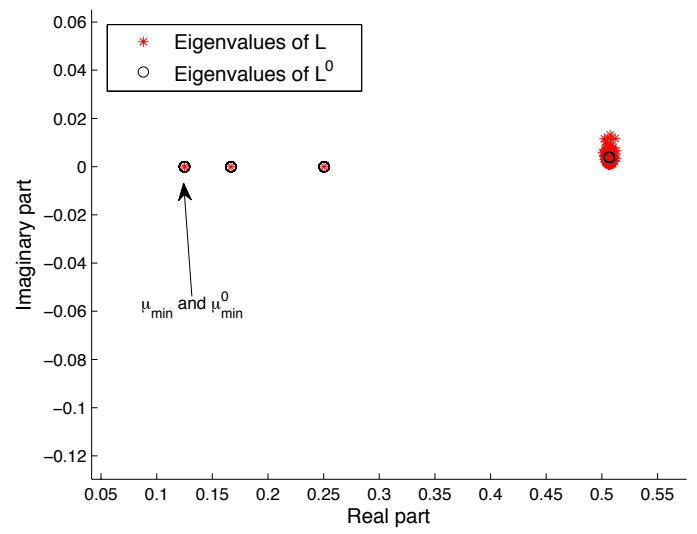

(b) Zoom around the eigenvalues associated with the modes $|m| \geq 1$.

Figure 6: Eigenvalues of the matrices $\mathbb{L}$ and $\mathbb{L}^{0}$, for $M=50$ unit disks $(k a=0.1$ and $k b \geq 10)$.

Let us begin by the spectral study of $\mathbb{L}^{0}$ by noticing that the matrix is almost diagonal since all the off-diagonal elements are zero except the ones with indices $(m, n)=(0,0)$ (see (18) and (21)). This implies that each coefficient $\left(\mathbb{L}^{0}\right)_{m, m}^{p, p}$ of the diagonal of $\mathbb{L}^{0}$ with index $m \neq 0$ is an eigenvalue of $\mathbb{L}^{0}$. Moreover, for $p, q=1, \ldots, M$ and $m \neq 0$, we have the following sequence of equalities

$$
\left(\mathbb{L}^{0}\right)_{-m,-m}^{p, p}=\left(\mathbb{L}^{0}\right)_{m, m}^{p, p}=\left(\mathbb{L}^{0}\right)_{m, m}^{q, q}=\left(\mathbb{L}^{0}\right)_{-m,-m}^{q, q}=\widehat{\mathbb{L}}_{|m|} .
$$


Hence, $\widehat{\mathbb{L}}_{m}$ is an eigenvalue of $\mathbb{L}^{0}$ with multiplicity $2 M$, for $m=1, \ldots, N$. We fix the following ordering, for $p=1, \ldots, M, m \neq 0$ and $-N \leq m \leq N$

$$
\left(\mu^{0}\right)_{m}^{p}=\widehat{\mathbb{L}}_{|m|}=\frac{a}{2|m|} .
$$

We know explicitly $2 N M$ eigenvalues of $\mathbb{L}^{0}$. Therefore, the computation of the $M$ last eigenvalues $\left(\left(\mu^{0}\right)_{0}^{p}\right)_{p=1, \ldots, M}$ can be made by using the smaller matrix built from the zero order modes (as done later). Let us now come back to the previous example. The approximation of the eigenvalues of $\mathbb{L}$ by the ones of $\mathbb{L}^{0}$ is reasonable for the eigenvalues related to the modes of order $m=0$ (Figure 6(a)) and for the higher order modes, $|m| \geq 2$ (Figure 6(b)). The approximation of the eigenvalues $\left(\mu_{1}^{p}\right)_{p=1, \ldots, M}$ associated with the first order modes are less accurate. This is probably due to the coupling between the propagative modes $m=0$ and the modes of order $m=1$. If the obstacles would be further, then this coupling can be expected weaker and the approximation better.

We now try to localize the eigenvalues $\mu_{\min }^{0}$ and $\mu_{\max }^{0}$ of $\mathbb{L}^{0}$ with smallest and largest modulii, respectively. To begin, we have the following ordering relations from (16)

$$
0<\widehat{\mathbb{L}}_{N}<\widehat{\mathbb{L}}_{N-1}<\ldots<\widehat{\mathbb{L}}_{2}<\widehat{\mathbb{L}}_{1} .
$$

Since $\widehat{\mathbb{L}}_{m}$, for $m=1, \ldots, N$, is an eigenvalue of $\mathbb{L}^{0}$ with multiplicity $2 M$, we have

$$
\left|\mu_{\text {min }}^{0}\right|=\min \left(\widehat{\mathbb{L}}_{N}, \min _{p=1, \ldots, M}\left|\left(\mu^{0}\right)_{0}^{p}\right|\right) .
$$

However, Figure 6 suggests that the $M$ eigenvalues $\left(\left(\mu^{0}\right)_{0}^{p}\right)_{p=1, \ldots, M}$ satisfy the inequality

$$
\left|\left(\mu^{0}\right)_{0}^{p}\right|>\widehat{\mathbb{L}}_{N} .
$$

When $k b_{p q} \rightarrow+\infty$, this relation can be proved [26] by using the Gershgorin disks for the matrix $\mathbb{L}^{0}$. Indeed, the off-diagonal coefficients $\left(\mathbb{L}^{0}\right)_{0,0}^{p, q}$ tend towards 0 when $k b_{p q} \rightarrow+\infty$ (see Equation (21)). Practically, this relation seems to be satisfied even when $k b_{p q}$ is not large enough to apply the approach based on the Gershgorin disks [26]. We therefore assume in the sequel that relation (26) holds. We estimate then $\mu_{\text {min }}^{0}$ by $\widehat{\mathbb{L}}_{N}$, i.e.

$$
\mu_{\text {min }}^{0}=\widehat{\mathbb{L}}_{N}=\frac{a}{2 N} .
$$

Since $\widehat{\mathbb{L}}_{N}$ is an eigenvalue with multiplicity $2 M$, then this is also the case for $\mu_{\text {min }}^{0}$. This conclusion confirms our study based on the Gershgorin disks realized in [26].

Now let us focus on the eigenvalue of $\mathbb{L}^{0}$ with largest modulus, denoted by $\mu_{\max }^{0}$. Figure 6 suggests that $\mu_{\max }^{0}$ is an eigenvalue related to the propagative modes with a modulus larger than the one which would come from the single scattering case. To prove these results and study more easily the $M$ eigenvalues $\left(\left(\mu^{0}\right)_{0}^{p}\right)_{p=1, \ldots, M}$, we build the smaller matrix $\mathbb{L}^{1}$ related to the modes of order 0 of $\mathbb{L}^{0}$. More precisely, $\mathbb{L}^{1}=\left(\mathbb{L}_{p, q}^{1}\right)_{1 \leq p, q \leq M}$ has size $M \times M$ and for all $1 \leq p, q \leq M$

$$
\mathbb{L}_{p, q}^{1}=\left(\mathbb{L}^{0}\right)_{0,0}^{p, q}= \begin{cases}\widehat{\mathbb{L}}_{0}=-a\left[\ln \left(\frac{k a}{2}\right)+\gamma\right]+i \frac{\pi a}{2} & \text { if } p=q, \\ \frac{i a \sqrt{\pi}}{\sqrt{2 k b_{p q}}} e^{i\left(k b_{p q}-\pi / 4\right)} & \text { otherwise. }\end{cases}
$$

The eigenvalues of $\mathbb{L}^{1}$ are then exactly $\left(\left(\mu^{0}\right)_{0}^{p}\right)_{p=1, \ldots, M}$, as it is proved in the following lemma. 
Lemma 2. The $M$ eigenvalues of $\mathbb{L}^{1}$ are exactly the $M$ eigenvalues $\left(\mu^{0}\right)_{0}^{p}$, for $p=1, \ldots, M$, of the matrix $\mathbb{L}^{0}$.

Proof. From relations (18) and (21), we can build two permutation matrices $\mathbb{P}$ and $\mathbb{Q}$ (that we do not explicit) such that

$$
\mathbb{P L}^{0} \mathbb{Q}=\left(\begin{array}{cc}
\mathbb{L}^{1} & 0 \\
0 & \mathbb{L}^{2}
\end{array}\right)
$$

where the matrix $\mathbb{L}^{1}$ is defined by $(28)$ and where the smaller matrix $\mathbb{L}^{2}:=\left(\left(\mathbb{L}^{2}\right)^{p, q}\right)_{1 \leq p, q \leq M}$, of size $2 N M \times 2 N M$, contains the diagonal of $\mathbb{L}^{0}$ without the modes of order 0 . More precisely, for $p=1, \ldots, M$, each diagonal block $\left(\mathbb{L}^{2}\right)^{p, p}$ has for coefficients, for $m=-N, \ldots, N, m \neq 0$,

$$
\left(\mathbb{L}^{2}\right)_{m, m}^{p, p}=\left(\mathbb{L}^{0}\right)_{m, m}^{p, p}=\widehat{\mathbb{L}}_{|m|}=\left(\mu^{0}\right)_{m}^{p},
$$

where we used the equality (24). Let us remark now that, since the matrix $\mathbb{L}^{2}$ is diagonal, its eigenvalues are also the diagonal coefficients, that is, from $(30),\left(\mu^{0}\right)_{m}^{p}$, for $p=1, \ldots, M, m=$ $-N, \ldots, N$ with $m \neq 0$. This implies that the eigenvalues of $\mathbb{L}^{1}$ must be $\left(\mu^{0}\right)_{0}^{p}$, for $p=1, \ldots, M$.

Let is now prove the following result.

Lemma 3. The following inequality holds

$$
\max _{p=1, \ldots, M}\left|\left(\mu^{0}\right)_{0}^{p}\right| \geq\left|\widehat{\mathbb{L}}_{0}\right| .
$$

Proof. The trace $\operatorname{tr}\left(\mathbb{L}^{1}\right)$ of $\mathbb{L}^{1}$ writes $\operatorname{tr}\left(\mathbb{L}^{1}\right)=\sum_{p=1}^{M} \widehat{\mathbb{L}}_{0}=M \widehat{\mathbb{L}}_{0}$. Moreover, it is also equal to the sum of the eigenvalues $\mathbb{L}^{1}$, leading to $M \widehat{\mathbb{L}}_{0}=\sum_{p=1}^{M}\left(\mu^{0}\right)_{0}^{p}$. By using the following inequality

$$
\left|M \widehat{\mathbb{L}}_{0}\right|=\left|\sum_{p=1}^{M}\left(\mu^{0}\right)_{0}^{p}\right| \leq \sum_{p=1}^{M}\left|\left(\mu^{0}\right)_{0}^{p}\right| \leq M \max _{p=1, \ldots, M}\left|\left(\mu^{0}\right)_{0}^{p}\right|,
$$

one finally gets $\left|\widehat{\mathbb{L}}_{0}\right| \leq \max _{p=1, \ldots, M}\left|\left(\mu^{0}\right)_{0}^{p}\right|$.

This Lemma leads to the following Proposition.

Proposition 1. The eigenvalue with largest modulus $\mu_{\text {max }}^{0}$ of $\mathbb{L}^{0}$ is an eigenvalue associated with the propagative modes $m=0$

$$
\mu_{\text {max }}^{0} \in\left\{\left(\mu^{0}\right)_{0}^{p}, p=1, \ldots, M\right\} .
$$

Furthermore, $\mu_{\text {max }}^{0}$ fulfills the inequality

$$
\left|\mu_{\max }^{0}\right| \geq\left|\widehat{\mathbb{L}}_{0}\right| .
$$

Proof. Since $|z| \geq|\Im(z)|$ for any complex number $z$, then, from (16), the following inequality holds $\left|\widehat{\mathbb{L}}_{0}\right| \geq \frac{a \pi}{2}$. Because $\widehat{\mathbb{L}}_{m}=a /(2 m)$ for $m=1, \ldots, N$, we have

$$
\left|\widehat{\mathbb{L}}_{0}\right|>\left|\widehat{\mathbb{L}}_{1}\right|>\left|\widehat{\mathbb{L}}_{2}\right|>\ldots>\left|\widehat{\mathbb{L}}_{N-1}\right|>\left|\widehat{\mathbb{L}}_{N}\right|
$$

From (24), we get $\left|\widehat{\mathbb{L}}_{0}\right|>\max _{1 \leq p \leq M, 1 \leq m \leq N}\left|\left(\mu^{0}\right)_{m}^{p}\right|$. Next, we apply Lemma 3 to obtain

$$
\max _{p=1, \ldots, M}\left|\left(\mu^{0}\right)_{0}^{p}\right| \geq\left|\widehat{\mathbb{L}}_{0}\right|>\max _{1 \leq p \leq M, 1 \leq m \leq N}\left|\left(\mu^{0}\right)_{m}^{p}\right|,
$$

which directly proves (32) and (33). 
This Proposition provides the following information. First, the eigenvalue with largest modulus for the multiple scattering problem $\mu_{\text {max }}^{0}$ must be found among the eigenvalues of $\mathbb{L}^{0}$ linked to the propagative modes $m=0$. Secondly, $\left|\mu_{\text {max }}^{0}\right|$ is larger than the modulus of the largest eigenvalue $\widehat{\mathbb{L}}_{0}$ for the single scattering case. By using Propostion 1 , the term $\left|\widehat{\mathbb{L}}_{0}\right|$ can be used to estimate $\left|\mu_{\max }^{0}\right|$. We propose another method leading to a sharper estimate of $\left|\mu_{\max }^{0}\right|$.

We do not have some explicit expressions of the $M$ eigenvalues of $\mathbb{L}^{1}$. Let us consider the example described in Figure 7 which displays the terms $\left(\widehat{\mathbb{L}}_{m}\right)_{0 \leq m \leq N}$ and the eigenvalues of $\mathbb{L}$, computed numerically. The medium is composed of 50 unitary disks randomly distributed in $[0,1000]^{2}$, in such a way that $k b \geq 10$ and $k a=0.1$, with $N=4$. We can observe that the $M=50$ eigenvalues $\left(\left(\mu^{0}\right)_{0}^{p}\right)_{p=1, \ldots, M}$ of $\mathbb{L}^{1}$ cluster around $\widehat{\mathbb{L}}_{0}(\simeq 2.4+1.6 i)$. This is related to the fact that the off-diagonal terms

$$
i a \sqrt{\frac{\pi}{2}} \frac{e^{i\left(k b_{p q}-\pi / 4\right)}}{\sqrt{k b_{p q}}},
$$

of $\mathbb{L}^{1}$ are highly oscillating and tend towards 0 when $k b_{p q} \rightarrow+\infty$. The eigenvalues are then complex perturbations of $\widehat{\mathbb{L}}_{0}$ (with a rotation around $\widehat{\mathbb{L}}_{0}$ ).

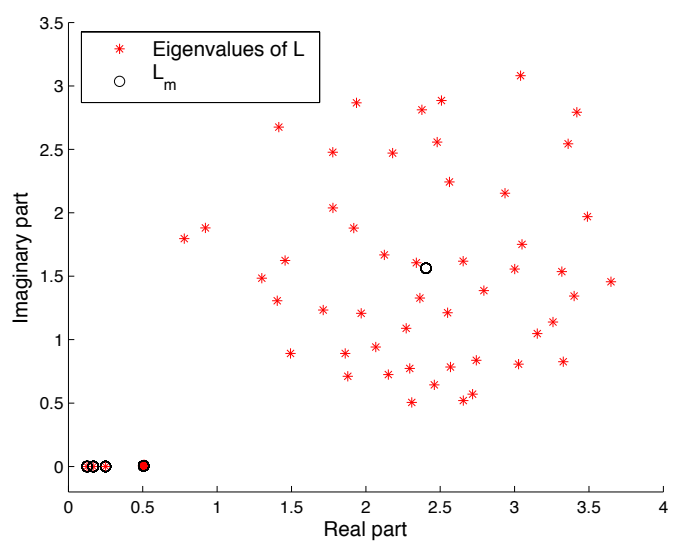

Figure 7: Eigenvalues of matrix $\mathbb{L}$ for $M=50$ unitary disks randomly distributed in $[0,1000]^{2}$, for $k a=0.1, k b \geq 10$ and $N=4$. The circles are associated with $\widehat{\mathbb{L}}_{m}$, for $m=0, \ldots, N$ and the stars to the eigenvalues of $\mathbb{L}$.

We propose now to apply the power iteration method to the matrix $\mathbb{L}^{1}$ to get an estimate of $\left|\mu_{\text {max }}^{0}\right|$. Let us introduce the uniform norm $\|\cdot\|_{\infty}: \forall \mathbf{X} \in \mathbb{C}^{M},\|\mathbf{X}\|_{\infty}=\max _{p=1, \ldots, M}\left|X_{p}\right|$. The first iteration leads to choose a normalized vector $\mathbf{X}$ and then to compute the matrix-vector product $\mathbf{Y}=\mathbb{L}^{1} \mathbf{X}$. The norm of vector $\mathbf{Y}$ is then an estimate of $\left|\mu_{\max }^{0}\right|$. Usually, one iteration is not sufficient to get an accurate approximation while this is the case here. Our choice of initial vector is $\mathbf{X}=(1,1, \ldots, 1)^{T}$. We then have: $\mathbf{Y}=\mathbb{L}^{1} \mathbf{X}=\left(Y_{1}, Y_{2}, \ldots, Y_{M}\right)^{T}$, with for $p=1, \ldots, M$

$$
Y_{p}=\widehat{\mathbb{L}}_{0}+\frac{i a \sqrt{\pi} e^{-i \pi / 4}}{\sqrt{2}} \sum_{1 \leq q \neq p \leq M} \frac{e^{i k b_{p q}}}{\sqrt{k b_{p q}}} .
$$


The uniform norm of $\mathbf{Y}$ is then an estimate of $\left|\mu_{\text {max }}^{0}\right|$

$$
\left|\mu_{\max }^{0}\right| \simeq \max _{p=1, \ldots, M}\left|Y_{p}\right|
$$

We now consider a similar situation to the one reported in Figure 7 (50 unit disks randomly distributed in $[0,1000]^{2}$, with $k a=0.1, k b \geq 10$ and $\left.N=4\right)$. We report on Figure 8 the coefficients of $\mathbf{Y}$ compared with the eigenvalues of $\mathbb{L}^{1}$. Let us remark that the coefficients $Y_{p}$ cluster around $\widehat{\mathbb{L}}_{0}$, like the eigenvalues of $\mathbb{L}^{1}$. We also note that the vector components of $\mathbf{Y}$ have a physical interpretation. Indeed, $\widehat{\mathbb{L}}_{0}$ represents the trace on the boundary $\Gamma_{p}$ of the Green's function $\frac{i}{4} H_{0}^{(1)}(\cdot)$ centered at $\mathbf{O}_{p}$ (up to a multiplicative constant). Furthermore, each term

$$
i \frac{e^{i k b_{p q}}}{\sqrt{k b_{p q}}} \frac{a \sqrt{\pi} e^{-i \pi / 4}}{\sqrt{2}}
$$

is the trace on $\Gamma_{p}$ of the far field Green's function centered at $\mathbf{O}_{q}$ (up to a multiplicative constant).

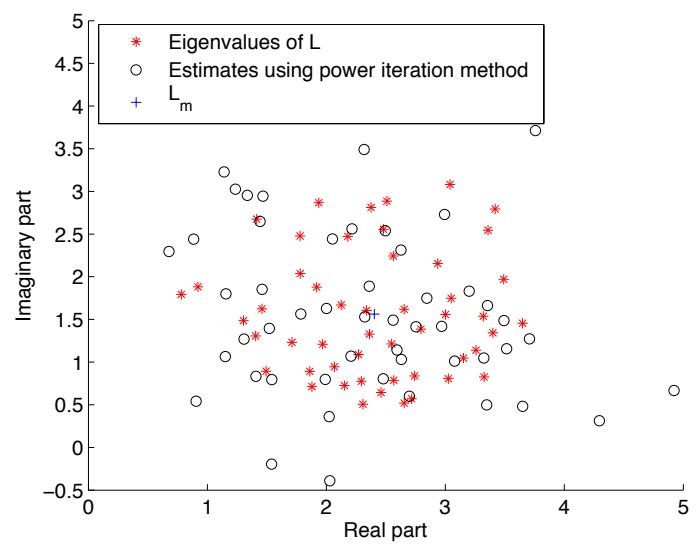

Figure 8: Comparison between the power iteration method (one iteration) and the eigenvalues of the matrix $\mathbb{L}^{1}$ for $M=50$ unit disks, for $k=0.1$ and $b \geq 100$.

The estimate (35) of $\left|\mu_{\text {max }}^{0}\right|$ provides an approximation of the eigenvalue with largest modulus $\left|\mu_{\text {max }}\right|$ of $\mathbb{L}$. Replacing $\widehat{\mathbb{L}}_{0}$ and $Y_{p}$ by their respective expressions in (35) one gets

$$
\left|\mu_{\max }\right| \simeq a \max _{p=1, \ldots, M}\left|-\left[\ln \left(\frac{k a}{2}\right)+\gamma\right]+i \frac{\pi}{2}+i \sqrt{\frac{\pi}{2}} e^{-i \pi / 4} \sum_{1 \leq q \neq p \leq M} \frac{e^{i k b_{p q}}}{\sqrt{k b_{p q}}}\right| .
$$

Let us remark that if we use the bound

$$
\left|\mu_{\max }\right| \leq a\left|-\left[\ln \left(\frac{k a}{2}\right)+\gamma\right]+i \frac{\pi}{2}\right|+\sqrt{\frac{\pi}{2}} \sum_{1 \leq q \neq p \leq M}\left|\frac{e^{i k b_{p q}}}{\sqrt{k b_{p q}}}\right|,
$$

we have the estimate obtained by the Gershgorin disks approach [26] which is not satisfactory as shown in Figure 9. As a consequence, we consider (36). Figure 9 compares $\left|\mu_{\max }\right|$ with the 
estimates obtained by the iterated power method (with one iteration), the approximation $\widehat{\mathbb{L}}_{0}$ of the eigenvalue with largest modulus for the single scattering case and the Gershgorin disks approach. The eigenvalue $\left|\mu_{\max }\right|$ is numerically computed by the eig Matlab function. The test case consists in launching 100 configurations for 50 unit disks randomly placed in $[0,1000]^{2}$. Here, we take $k b \geq 10$ and $k a=0.1$. We observe that the power iteration method (in this example) provides an over estimate of $\left|\mu_{\max }\right|$ which is sharper than the maximal eigenvalue $\left|\widehat{\mathbb{L}}_{0}\right|$ of the single scattering problem. The average relative error is $19 \%$. Finally, the estimate based on the Gershgorin disks is clearly not satisfactory.

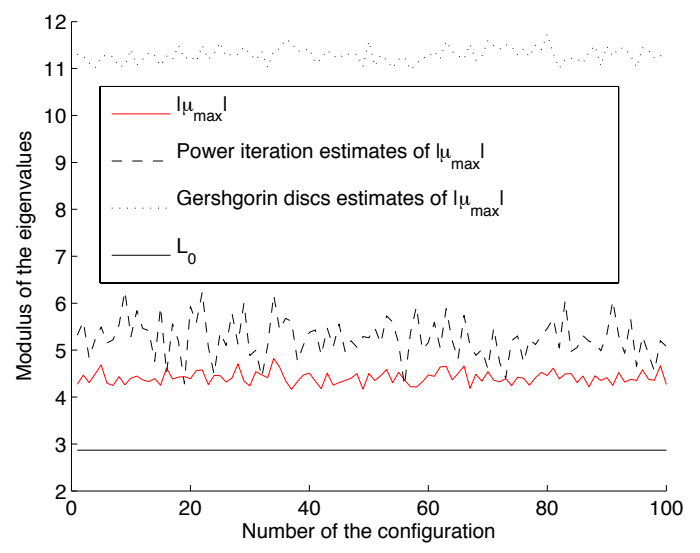

Figure 9: Comparison between $\left|\mu_{\max }\right|$, the power iteration method (one iteration), $\widehat{\mathbb{L}}_{0}$ and the estimate based on the Gershgorin disks, for 100 configurations made of $M=50$ unit disks randomly distributed in $[0,1000]^{2}$, with $k=0.1, N=4$ and $b \geq 100$.

We assume now that the matrix is almost normal and that the quantity

$$
\frac{\left|\mu_{\max }\right|}{\left|\mu_{\min }\right|}
$$

estimates the condition number $\operatorname{cond}_{2}(\mathbb{L})$. Replacing $\left|\mu_{\text {min }}\right|$ and $\left|\mu_{\text {max }}\right|$ by their respective approximation (27) and (36) in (37) leads to the approximate condition number $\operatorname{cond}_{a p p}(\mathbb{L})$

$$
\operatorname{cond}_{a p p}(\mathbb{L})=2 N \max _{p=1, \ldots, M}\left|-\left[\ln \left(\frac{k a}{2}\right)+\gamma\right]+i \frac{\pi}{2}+i \sqrt{\frac{\pi}{2}} e^{-i \pi / 4} \sum_{1 \leq q \neq p \leq M} \frac{e^{i k b_{p q}}}{\sqrt{k b_{p q}}}\right| .
$$

One example of conditioning is reported in Figure 10(a), for 100 configurations made of 50 unit disks randomly placed in $[0,1000]^{2}$, with $k b \geq 10$ and $k a=0.1$. The obtained estimate leads to a relative error equal to $7 \%$ which is relatively acceptable for such a difficult problem.

\subsection{Link with the boundary element approximation and formal extensions to other objects}

For any $p=1, \ldots, M$, we denote by $\Gamma_{p, h}=\cup_{j=1}^{N_{p, h}} K_{p, j}$. a polygonal approximation of the smooth curve $\Gamma_{p}$, using $N_{p, h}$ segments $K_{p, j}, 1 \leq j \leq N_{p, h}$. We designate by $h_{p, j}$ the length of $K_{p, j}$ and by 


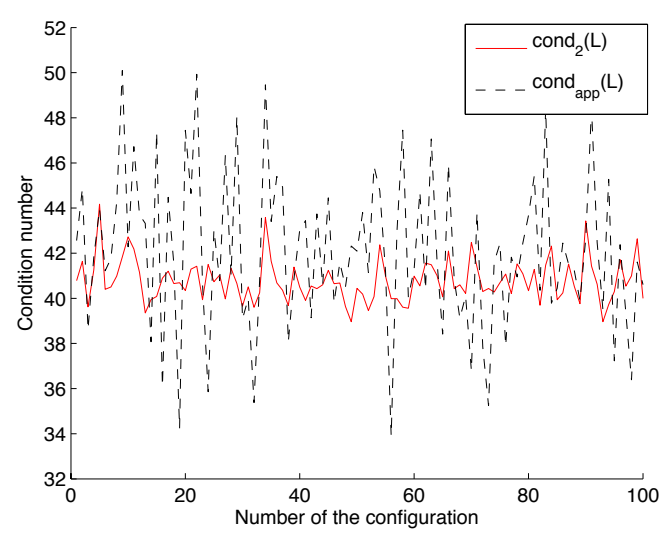

(a) Comparison between the exact and approximate condition number

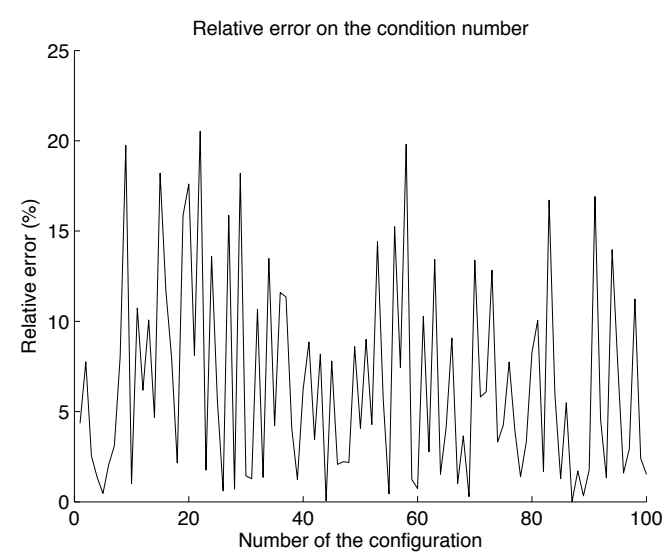

(b) Relative error on the conditioning (\%) : $100\left|\frac{\operatorname{cond}(\mathbb{L})-\operatorname{cond}_{a p p}(\mathbb{L})}{\operatorname{cond}(\mathbb{L})}\right|$

Figure 10: Comparison between the exact and approximate condition numbers of $\mathbb{L}$, for 100 configurations with $M=50$ randomly distributed unit disks in $[0,1000]^{2}(k=0.1, N=4$ and $b \geq 100)$.

$h$ the largest length $h=\max _{1 \leq p \leq M}\left(\max _{1 \leq j \leq N_{p, h}} h_{p, j}\right)$. We choose the boundary element subspace $V_{h} \subset L^{2}\left(\Gamma_{h}\right)$ as $V_{h}:=\left\{\rho_{h} \in \mathscr{C}^{0}\left(\Gamma_{h}\right)\right.$ such that $\left.\rho_{h}\right|_{K_{p, j}} \in \mathbb{P}_{1}$, with $1 \leq p \leq M$ and $\left.1 \leq j \leq N_{p, h}\right\}$. We then have to estimate the eigenvalues of $\left[M_{h}\right]^{-1}\left[L_{h}\right]$, where $N_{t o t, h}=\sum_{p=1}^{M} N_{p, h}$ is the total number of segments, $\left[L_{h}\right] \in \mathcal{M}_{N_{t o t, h}, N_{t o t, h}}(\mathbb{C})$ is the single-layer potential matrix and $\left[M_{h}\right] \in$ $\mathcal{M}_{N_{t o t, h}, N_{\text {tot }, h}}(\mathbb{C})$ is the mass matrix for linear boundary elements. In the sequel, we denote by $\mu_{\text {min }}^{h}$ and $\mu_{\text {max }}^{h}$ the eigenvalue with smallest and largest modulus of the matrix $\left[M_{h}\right]^{-1}\left[L_{h}\right]$, respectively.

\subsubsection{The circular cylinder}

To link the spectral Fourier approximation to the boundary element method, we begin by considering circular cylinders with radius $a$. The circles $\Gamma_{p}$ are uniformly meshed by $N_{h}=N_{p, h}$ segments of length $h$, for $p=1, \ldots, M$. The number of degrees of freedom $N_{E}$ of the boundary element method to discretize $\Omega_{p}$ is $N_{E}=N_{h} \simeq \frac{2 \pi a}{h}$. This must be compared with the $2 N+1$ modes used for the spectral Fourier method. By formally substituting $N$ by $\pi a h^{-1}-1 / 2$ in the estimates (27) and (36) respectively of $\mu_{\min }$ and $\left|\mu_{\max }\right|$, one gets $\mu_{\min }^{h} \simeq \mu_{\min }^{a p p}(a, h)$ and $\left|\mu_{\max }^{h}\right| \simeq\left|\mu_{\max }^{a p p}(a, k)\right|$, when $k a$ tends towards zero and $k b$ towards infinity, with

$$
\left\{\begin{array}{l}
\mu_{\min }^{a p p}(a, h)=\frac{a}{2 \pi a h^{-1}-1}, \\
\left|\mu_{\text {max }}^{a p p}(a, k)\right|=a \max _{p=1, \ldots, M}\left|-\left[\ln \left(\frac{k a}{2}\right)+\gamma\right]+i \frac{\pi}{2}+i \sqrt{\frac{\pi}{2}} e^{-i \pi / 4} \sum_{1 \leq q \neq p \leq M} \frac{e^{i k b_{p q}}}{\sqrt{k b_{p q}}}\right| .
\end{array}\right.
$$

We then obtain the following spectral condition number estimate (38)

$$
\operatorname{cond}\left(k, a, \Gamma_{h}\right):=\operatorname{cond}_{2}\left(\left[M_{h}\right]^{-1}\left[L_{h}\right]\right) \simeq \operatorname{cond}_{a p p}(k, a, h),
$$


with

$$
\begin{aligned}
& \operatorname{cond}_{a p p}(k, a, h)=\left|\frac{\mu_{\text {max }}^{a p p}(a, h)}{\mu_{\text {min }}^{a p p}(a, k)}\right| \\
& \quad=\left(2 \pi a h^{-1}-1\right) \max _{p=1, \ldots, M}\left|-\left[\ln \left(\frac{k a}{2}\right)+\gamma\right]+i \frac{\pi}{2}+\sqrt{\frac{\pi}{2}} e^{-i \pi / 4} \sum_{1 \leq q \neq p \leq M} \frac{e^{i k b_{p q}}}{\sqrt{k b_{p q}}}\right| .
\end{aligned}
$$

We validate the approximation (40) on Figure 11 by considering the previous geometrical parameters $\left(M=50\right.$ unit disks randomly placed in $\left.[0,1000]^{2}, k b \geq 10, k a=0.1\right)$. Each obstacle is meshed with $N_{h}=50$ segments. For 100 configurations, we compute the eigenvalues of the matrix $\left[M_{h}\right]^{-1}\left[L_{h}\right]$ with smallest and largest modulii as well as its condition number. From Figures 11(a)-11(b), we observe that one gets a satisfactory mean relative error on $\mu_{\text {min }}^{h}$ equal to $2.5 \%$. Figure 11(c) shows that the estimate of $\left|\mu_{\text {max }}^{h}\right|$ is of the same order as $\left|\mu_{\max }^{h}\right|$. The relative error is much more important and equal to about $19 \%$ in average. However, the estimate obtained for the condition number is relatively accurate, with a mean relative error equal to $7 \%$ (Figures 11(e)-11(f)).

\subsubsection{Extensions to other geometries}

Like for single scattering case (Section 2.2), we try to formally extend our estimates to elliptical and rectangular cylinders. To this end, we have to replace the radius $a$ and the mesh step $h$ respectively by an equivalent radius $a_{\text {eqv }}$ and an equivalent mesh step $h_{\text {eqv }}$ in the eigenvalues estimates.

For the ellipse, we proposed the following three equivalent radii

$$
a_{\mathrm{eqv}}^{1}=\frac{a_{x_{1}}+a_{x_{2}}}{2}, \quad a_{\mathrm{eqv}}^{2}=\frac{2 a b}{a_{x_{1}}+a_{x_{2}}}, \quad a_{\mathrm{eqv}}^{3}=\frac{\sqrt{a_{x_{1}}^{2}+a_{x_{2}}^{2}}}{\sqrt{2}} .
$$

Concerning the equivalent mesh size $h_{\text {eqv }}$, the smallest discretization step provided the best estimate of $\mu_{\text {min }}^{h}$. We then set $h_{\text {eqv }}=\min _{1 \leq p \leq M} \min _{1 \leq j \leq N_{h, p}} h_{p, j}$. To get an estimate of the eigenvalues $\mu_{\text {min }}^{h}$ and $\left|\mu_{\text {max }}^{h}\right|$, we formally replace $a$ by an equivalent radius $a_{\mathrm{eqv}}^{j}$, for $j=1,2$ or 3 , and the mesh size $h$ by $h_{\text {eqv }}$, into (39). This substitution is also considered in the expression (40) of $\operatorname{cond}_{a p p}(a, h, k)$ to estimate the condition number of $\left[M_{h}\right]^{-1}\left[L_{h}\right]$. We validate and compare these estimates for different equivalent radius by considering $M=50$ ellipses with semi-axes $a_{x_{1}}=1$ and $a_{x_{2}}=0.25$ randomly distributed in $[0,1000]^{2}$. We take $k=0.1$ and $b \geq 100$, where $b$ represents the smallest distance between the centers of two ellipses. For 100 configurations, we numerically compare on Figure 12 the eigenvalues of the matrix $\left[M_{h}\right]^{-1}\left[L_{h}\right]$ with their estimates as well as the exact and estimated condition numbers. The numerical simulations show that the equivalent radius $a_{\text {eqv }}^{2}$ gives inaccurate estimates of $\left|\mu_{\max }\right|$. For clarity, we only consider the results for $a_{\text {eqv }}^{1}$ and $a_{\text {eqv }}^{3}$. Let us begin by studying the estimate of $\mu_{\min }^{h}$ reported on Figures 12(a)-12(b). The relative errors are of the same order $(19 \%)$ for the two equivalent radii $a_{\text {eqv }}^{1}$ and $a_{\text {eqv }}^{3}$. This is more important than for the circular case. This can be explained by the fact that the eigenvalue with smallest modulus strongly depends on the minimal mesh size. For an ellipse (and most particularly if it is thin), the mesh step can be locally small most particularly when the curvature is strong. An accurate estimate of $\mu_{\min }^{h}$ is then more difficult to obtain. Concerning the modulus of $\mu_{\text {max }}^{h}$, we observe on Figures $12(\mathrm{c})-12(\mathrm{~d})$ that the two estimates are relatively correct. We can notice that the radius $a_{\text {eqv }}^{1}$ 


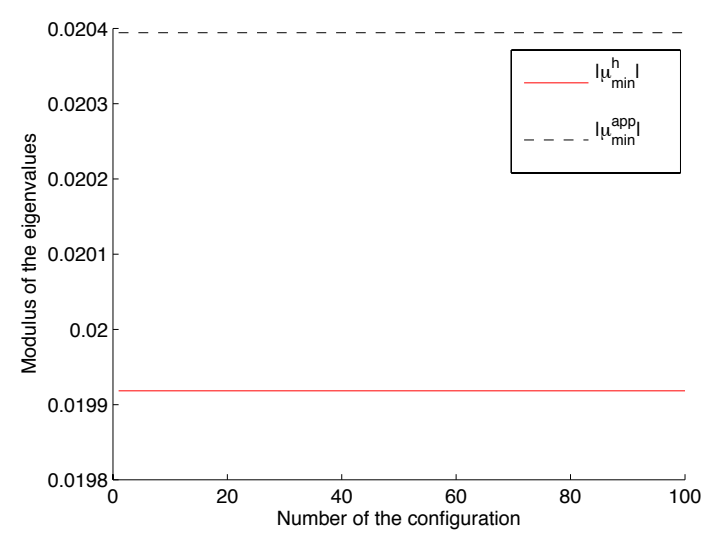

(a) Comparison between the eigenvalue with smallest modulus and it estimate (39)

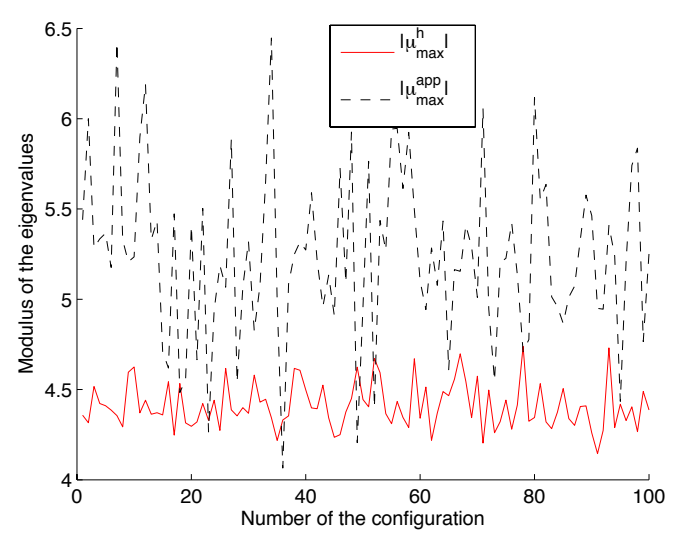

(c) Comparison between the eigenvalue with largest modulus and its estimate (39)

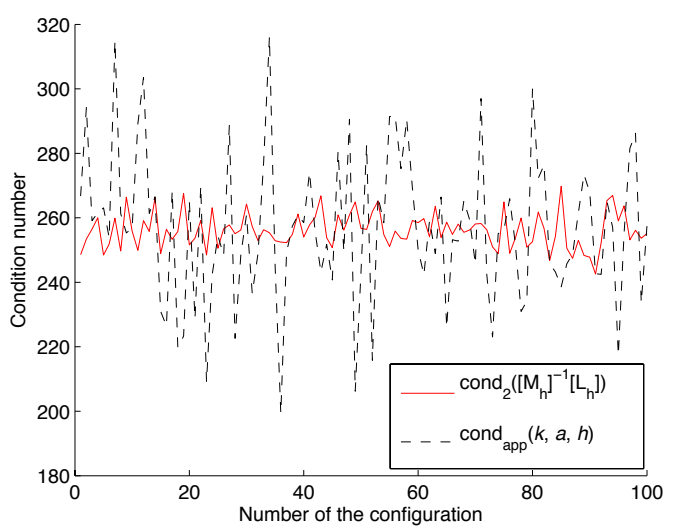

(e) Comparison between the exact and approximate condition numbers

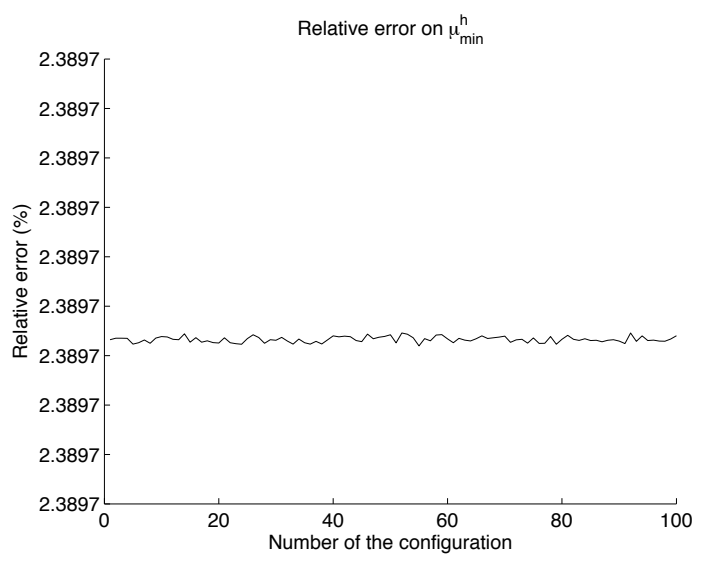

(b) Relative error on $\left|\mu_{\text {min }}^{h}\right|$

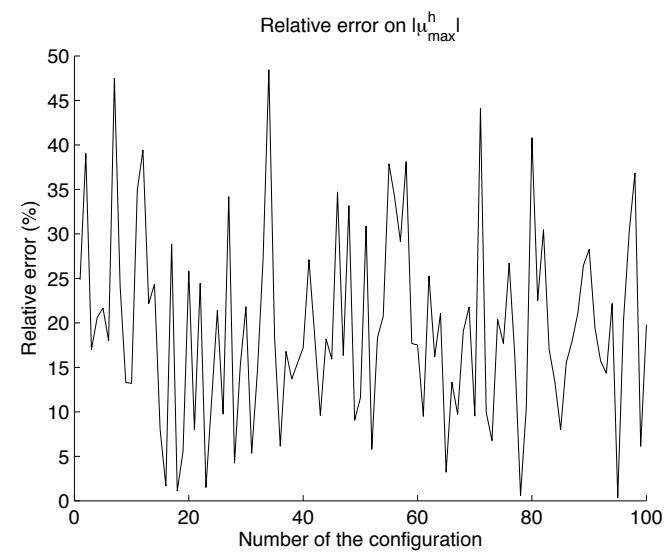

(d) Relative error on $\left|\mu_{\max }^{h}\right|$

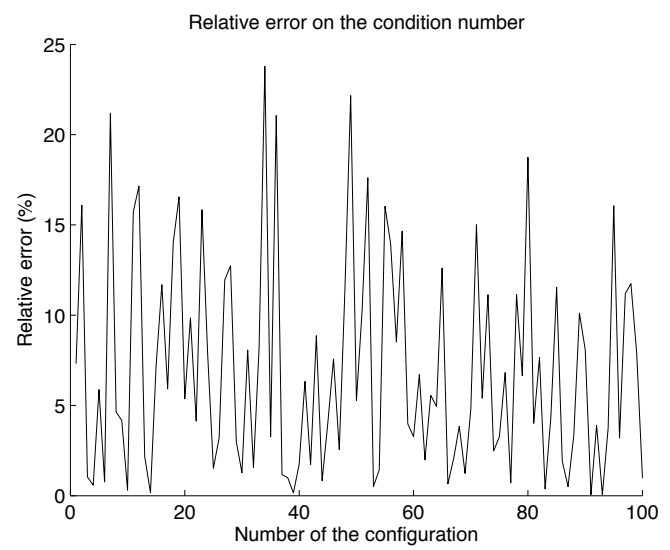

(f) Relative error on the condition number

Figure 11: Comparison of the estimates (39) and (40) of the modulus of the eigenvalues with smallest and largest modulii as well as condition number of the matrix $\left[M_{h}\right]^{-1}\left[L_{h}\right]$. The obstacles are circular cylinders discretized by using $N_{h}=50$ segments. For each of the 100 configurations, $M=50$ unitary disks are randomly distributed in $[0,1000]^{2}$, with $k=0.1$ and $b \geq 100$. 
leads to a better estimate than $a_{\text {eqv }}^{3}$ unlike the single scattering case. More precisely, the relative error on $\left|\mu_{\text {max }}^{h}\right|$ is about $9 \%$ in average for the equivalent radius $a_{\text {eqv }}^{1}$, which is of the same order as for circular cylinders, and about $22 \%$ for the equivalent radius $a_{\text {eqv }}^{3}$. Finally, we compare on Figures $12(\mathrm{e})$ and $12(\mathrm{f})$ the condition number of the matrix $\left[M_{h}\right]^{-1}\left[L_{h}\right]$ with its estimate. We have only reported the results related to $a_{\text {eqv }}^{1}$ which lead to the best approximation of $\left|\mu_{\text {max }}^{h}\right|$. The relative error on the condition number is then about $11 \%$, which is satisfactory.

To end up, we adapt the previous estimates to rectangular cylinders. For one rectangle with half size lengths $a_{x_{1}}$ and $a_{x_{2}}$, we proposed to take the equivalent radius $a_{\text {eqv }}^{4}$ (13) given by

$$
a_{\mathrm{eqv}}^{4}=\frac{(1+\sqrt{2})}{2} \frac{\sqrt{a_{x_{1}}^{2}+a_{x_{2}}^{2}}}{\sqrt{2}} .
$$

The equivalent step $h_{\text {eqv }}$ is always chosen as the smallest mesh size

$$
h_{\mathrm{eqv}}=\min _{1 \leq p \leq M} \min _{1 \leq j \leq N_{h, p}} h_{p, j} .
$$

We consider the following numerical example: $M=50$ rectangles with half size lengths $a_{x_{1}}=1$ and $a_{x_{2}}=0.25$, randomly distributed in $[0,1000]^{2}$. The wave number $k$ is 0.1 and the smallest distance $b$ between the centers of two rectangles is equal to 100. Each rectangle is meshed with $N_{h}=48$ segments (12 segments by side). We numerically compute the condition number of the matrix $\left[M_{h}\right]^{-1}\left[L_{h}\right]$ as well as its estimate (40) for 100 configurations (Figure 13). As shown on Figures 13(a) and 13(b), the estimate of the eigenvalue with smallest modulus is very accurate since the relative error is about $2 \%$. This is probably due to the fact that the curvature on each side is equal to zero even if the mesh is globally non uniform (but the mesh step is constant on each side). Furthermore, the estimate of the modulus of $\mu_{\max }^{h}$ leads to a relative error of $27 \%$, (Figures 13(c)13(d)). This error is larger than for the case of ellipses or disks. Finally, the condition number estimate is accurate with a relative error equal to $7 \%$ (Figures $13(\mathrm{e})-13(\mathrm{f})$ ). Hence, the formal extension to ellipses and rectangles is satisfactory for computing estimates of the condition number of the matrix $\left[M_{h}\right]^{-1}\left[L_{h}\right]$ resulting from a linear boundary element discretization of the single-layer potential in the framework of low-frequency multiple scattering with relatively distant obstacles.

\section{Conclusion}

In this paper, we have presented some asymptotic spectral and condition number estimates of the acoustic single-layer potential for low frequency multiple scattering problems. This first study assumes that the obstacles are far enough (dilute media) to derive the approximate formulas. We have shown in [26] that an approach based on the Gershgorin disks remains limited. For this reason, we propose here an alternative approach that provides better estimates. All these approximations are validated in the case of circular cylinders and formally extended to elliptical and rectangular cylinders discretized by using linear boundary element methods with non uniform meshes. In [7], we derive new estimates for the case of close obstacles by using the approach derived here and new asymptotics. Furthermore, let us note that a similar analysis should be possible for the threedimensional case since the two-dimensional strategy can be extended. However, this requires to write all the technical details which is out of the scope of the paper. 


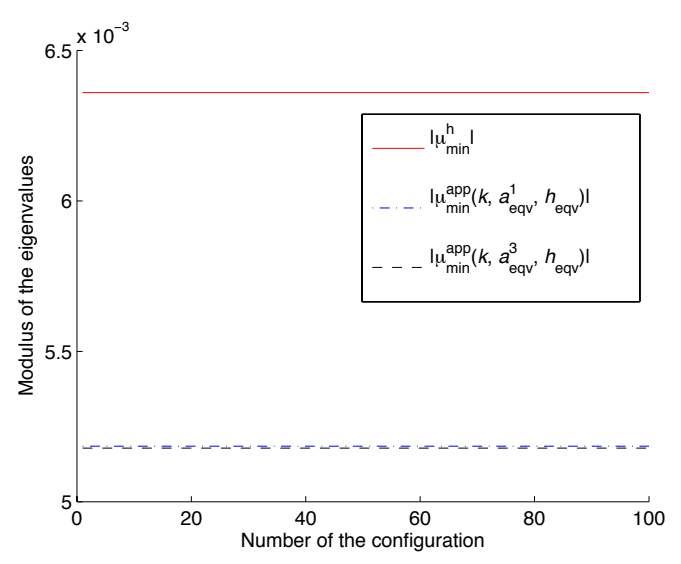

(a) Comparison between the eigenvalue with smallest modulus and its estimate

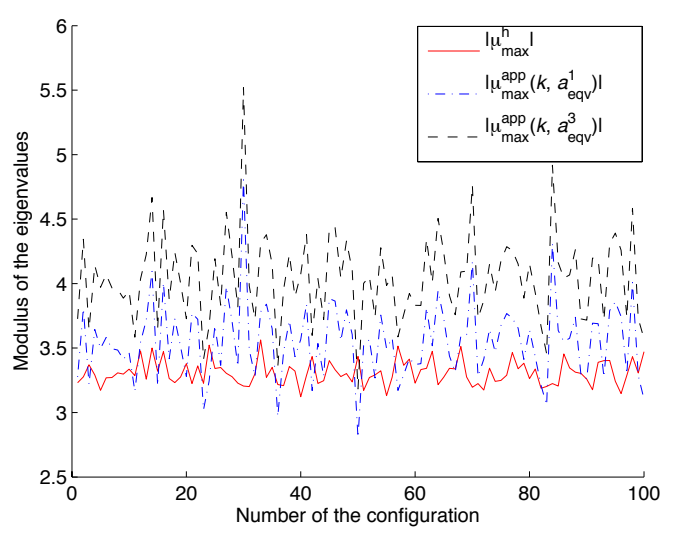

(c) Comparison between the eigenvalue with largest modulus and its estimate

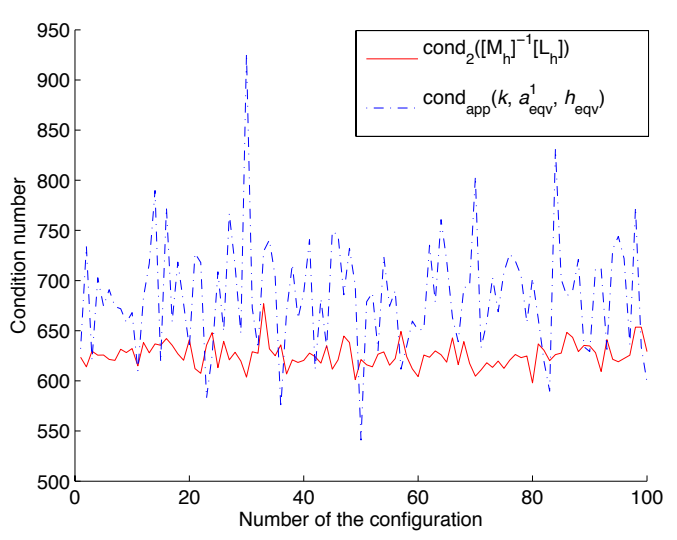

(e) Comparison between the exact and approximate condition numbers

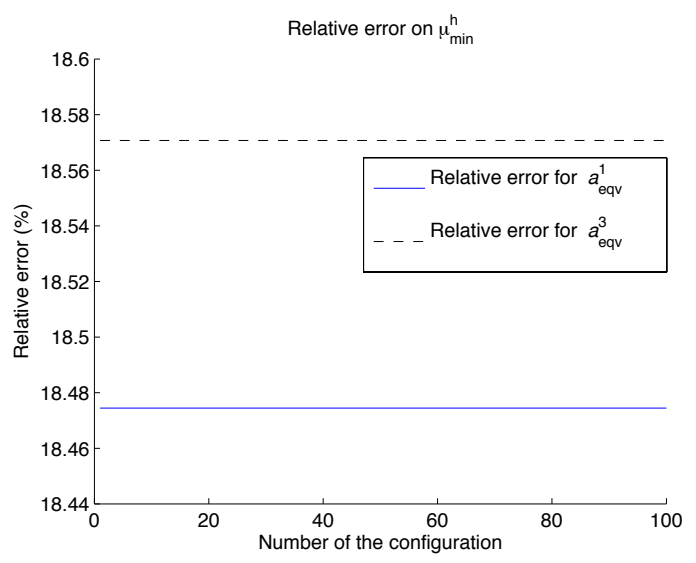

(b) Relative error on $\left|\mu_{\text {min }}^{h}\right|$

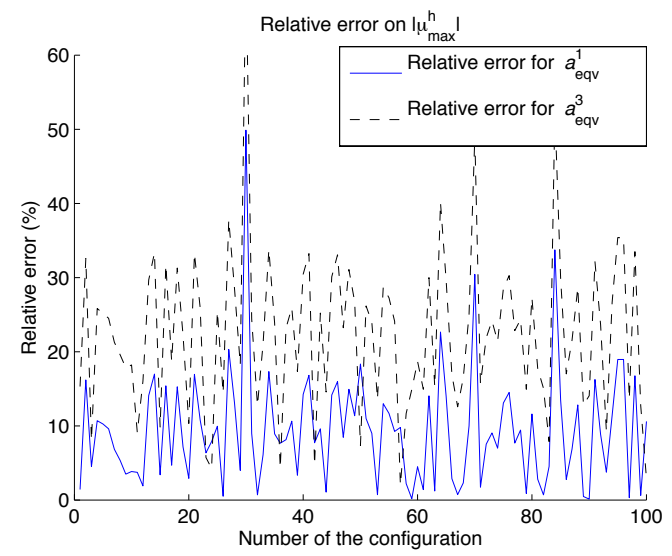

(d) Relative error on $\left|\mu_{\max }^{h}\right|$

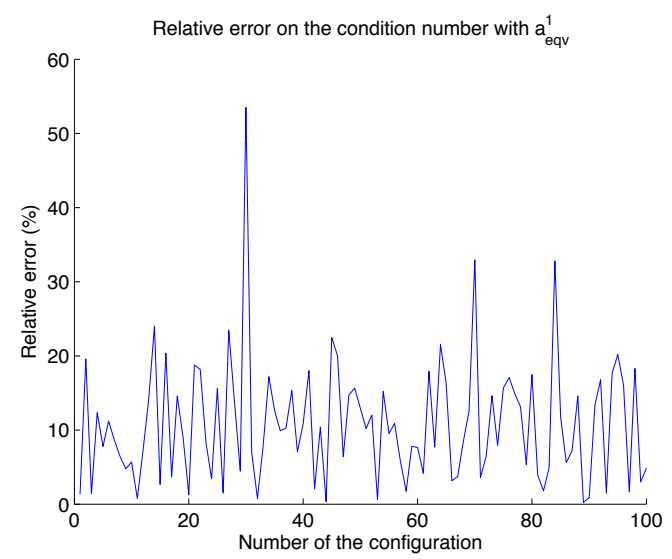

(f) Relative error on the condition number

Figure 12: Comparison of the estimates of the eigenvalues with smallest and largest modulii as well as condition number of the matrix $\left[M_{h}\right]^{-1}\left[L_{h}\right]$ for elliptical cylinders with semi-axes $a_{x_{1}}=1$ and $a_{x_{2}}=0.25$. Each ellipse is discretized by using $N_{h}=50$ segments. For each of the 100 configurations, $M=50$ ellipses are randomly distributed in $[0,1000]^{2}$, with $k=0.1$ and $b \geq 100$. 


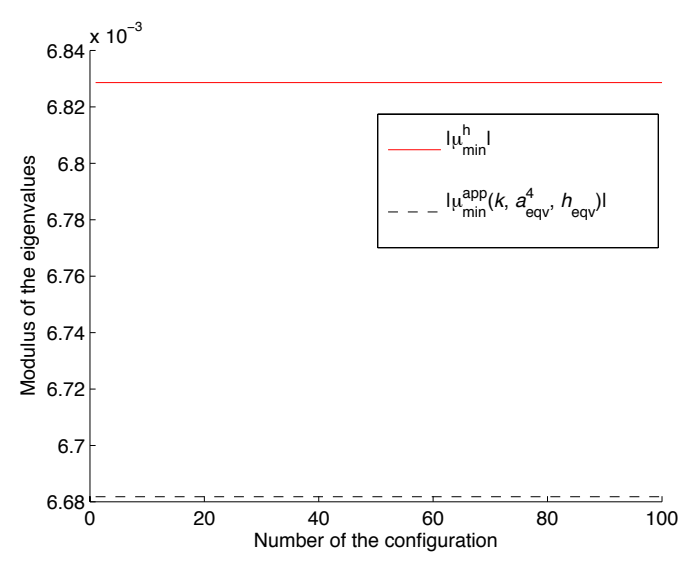

(a) Comparison between the eigenvalue with smallest modulus and its estimate

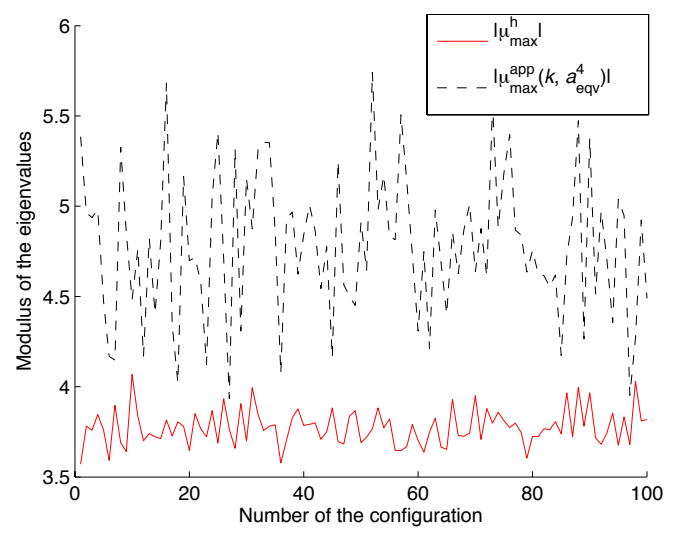

(c) Comparison between the eigenvalue with largest modulus and its estimate

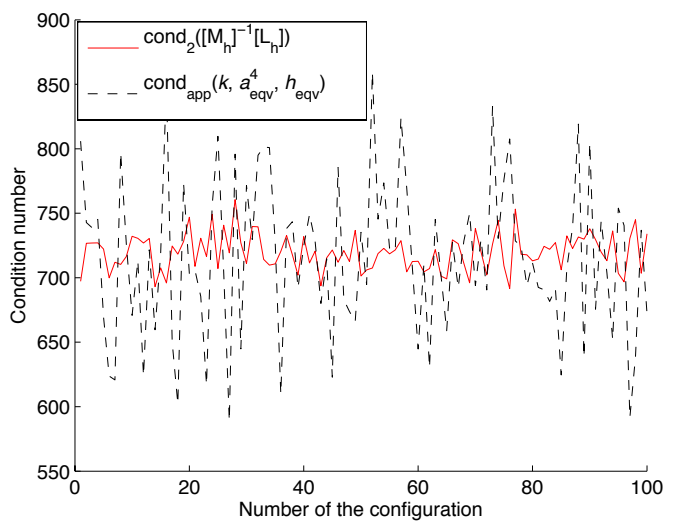

(e) Comparison between the exact and approximate condition numbers

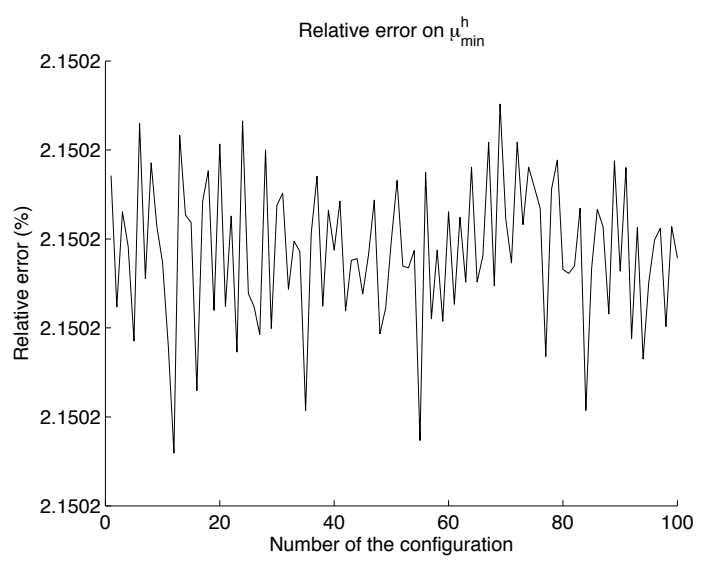

(b) Relative error on $\left|\mu_{\text {min }}^{h}\right|$

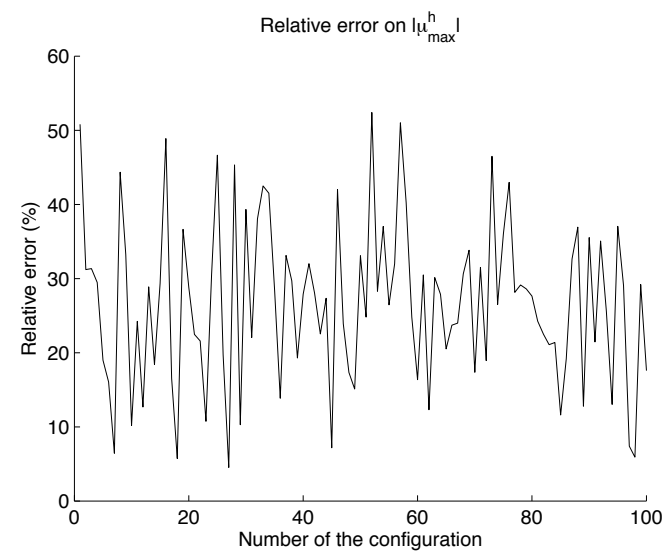

(d) Relative error on $\left|\mu_{\max }^{h}\right|$

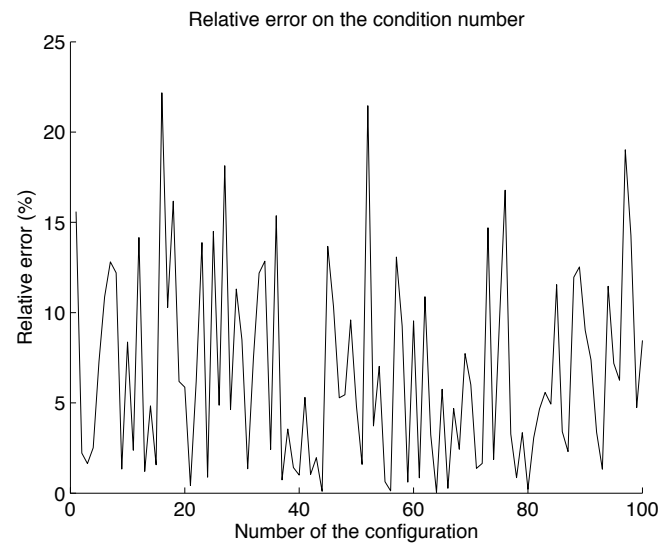

(f) Relative error on the condition number

Figure 13: Comparison of the estimates of the eigenvalues with smallest and largest modulii as well as condition number of the matrix $\left[M_{h}\right]^{-1}\left[L_{h}\right]$ for rectangular cylinders with half side lengths $a_{x_{1}}=1$ and $a_{x_{2}}=0.25$. Each rectangle is discretized by using $N_{h}=48$ segments. For each of the 100 configurations, $M=50$ rectangular cylinders are randomly distributed in $[0,1000]^{2}$, with $k=0.1$ and $b \geq 100$. 


\section{References}

[1] M. Abramowitz and I.A. Stegun. Handbook of Mathematical Functions with Formulas, Graphs, and Mathematical Tables. New York: Dover Publiations, Ninth Printing (1972).

[2] X. Antoine, A. Bendali, and M. Darbas. Analytic preconditioners for the electric field integral equation. Internat. J. Numer. Methods Engrg., (61):1310-1331, 2004.

[3] X. Antoine, A. Bendali, and M. Darbas. Analytic preconditioners for the boundary integral solution of the scattering of acoustic waves by open surfaces. J. Comput. Acoust., 3(13):477498, 2005.

[4] X. Antoine, C. Chniti, and K. Ramdani. On the numerical approximation of high-frequency acoustic multiple scattering problems by circular cylinders. J. Comput. Phys., 227(3):1754$1771,2008$.

[5] X. Antoine and M. Darbas. Alternative integral equations for the iterative solution of acoustic scattering problems. Quaterly J. Mech. Appl. Math., 1(58):107-128, 2005.

[6] X. Antoine and M. Darbas. Generalized combined field integral equations for the iterative solution of the three-dimensional Helmholtz equation. M2AN Math. Model. Numer. Anal., $1(41): 147-167,2007$.

[7] X. Antoine and B. Thierry. Spectral and condition number estimates of the acoustic single-layer operator for low frequency multiple scattering in dense media. submitted, 2011.

[8] A. Bendali and M. Fares. Boundary integral equations methods in acoustics in computational acoustic scattering. In Computational Methods for Acoustics Problems, pages 1-36. SaxeCoburg Publications, 2008.

[9] T. Betcke, S.N. Chandler-Wilde, I.G. Graham, S. Langdon, and M. Lindner. Condition number estimates for combined potential operators in acoustics and their boundary element discretisation. Numer. Methods Partial Differential Eq., to appear, 2011.

[10] H. Brakhage and P. Werner. Über das Dirichletsche Aussenraumproblem für die Helmholtzsche Schwingungsgleichung. Arch. Math., 16:325-329, 1965.

[11] S.N. Chandler-Wilde, I.G. Graham, S. Langdon, and M. Lindner. Condition number estimates for combined potential boundary integral operators in acoustic scattering. J. Integral Equations Appl., (21):229-279, 2009.

[12] J. T. Chen, J. H. Lin, S. R. Kuo, and S. W. Chyuan. Boundary element analysis for the Helmholtz eigenvalue problems with a multiply connected domain. R. Soc. Lond. Proc. Ser. A Math. Phys. Eng. Sci., 457(2014):2521-2546, 2001.

[13] W.C. Chew, J.M. Jin, E. Michielssen, and J. Song. Fast and Efficient Algorithms in Computational Electromagnetics. Artech House Antennas and Propagation Library, Norwood, 2001.

[14] W.C. Chew and K.F. Warnick. On the spectrum of the electric field integral equation and the convergence of the moment method. Internat. J. Numer. Methods Engrg., (51):475-489, 2001. 
[15] D. L. Colton and R. Kress. Integral Equation Methods in Scattering Theory. Pure and Applied Mathematics (New York). John Wiley \& Sons Inc., New York, 1983. A Wiley-Interscience Publication.

[16] M. Darbas. Préconditionneurs Analytiques de type Calderòn pour les Formulations Intégrales des Problèmes de Diffraction d'Ondes. PhD thesis, INSA de Toulouse, 2004.

[17] L. Greengard and V. Rokhlin. A fast algorithm for particle simulations. J. Comput. Phys., $73(2): 325-348,1987$.

[18] M.J. Grote and C. Kirsch. Dirichlet-to-Neumann boundary conditions for multiple scattering problems. J. Comput. Phys., (201):630-650, 2004.

[19] R.F. Harrington and J.R. Mautz. H-field, E-field and combined field solution for conducting bodies of revolution. Archiv Elektronik und Uebertragungstechnik, 4(32):157-164, 1978.

[20] R. Kress. Minimizing the condition number of boundary integral operators in acoustic and electromagnetic scattering. Quart. J. Mech. Appl. Math., 38(2):323-341, 1985.

[21] R. Kress and W. T. Spassov. On the condition number of boundary integral operators for the exterior Dirichlet problem for the Helmholtz equation. Numer. Math., 42(1):77-95, 1983.

[22] P. A. Martin. Multiple Scattering. Interaction of Time-Harmonic Waves with N Obstacles, volume 107 of Encyclopedia of Mathematics and its Applications. Cambridge University Press, Cambridge, 2006.

[23] J.-C. Nédélec. Acoustic and Electromagnetic Equations. Integral Representations for Harmonic Problems, volume 144 of Applied Mathematical Sciences. Springer-Verlag, New York, 2001.

[24] Y. Saad. Iterative Methods for Sparse Linear Systems. SIAM, (2nd edition) 2003.

[25] Y. Saad and M. Schultz. GMRES: a generalized minimal residual algorithm for solving nonsymmetric linear systems. SIAM J. Sci. Statist. Comput., 7(3):856-869, 1986.

[26] B. Thierry. Analyse et Simulations Numériques du Retournement Temporel et de la Diffraction Multiple. Nancy University, 2011. 